\title{
Predictive simulation of guide-wave structural health monitoring
}

\section{Victor Giurgiutiu}

Victor Giurgiutiu, "Predictive simulation of guide-wave structural health monitoring," Proc. SPIE 10170, Health Monitoring of Structural and Biological Systems 2017, 1017002 (28 April 2017); doi: 10.1117/12.2263325 Health Monitoring, 2017, Portland, Oregon, United States 


\title{
Predictive Simulation of Guide-Wave Structural Health Monitoring
}

\author{
Victor Giurgiutiu \\ Mechanical Engineering Dept., University of South Carolina \\ 300 S. Main St., Columbia, SC 29208, USA, victorg@sc.edu
}

\begin{abstract}
This paper presents an overview of recent developments on predictive simulation of guided wave structural health monitoring (SHM) with piezoelectric wafer active sensor (PWAS) transducers. The predictive simulation methodology is based on the hybrid global local (HGL) concept which allows fast analytical simulation in the undamaged global field and finite element method (FEM) simulation in the local field around and including the damage. The paper reviews the main results obtained in this area by researchers of the Laboratory for Active Materials and Smart Structures (LAMSS) at the University of South Carolina, USA. After thematic introduction and research motivation, the paper covers four main topics: (i) presentation of the HGL analysis; (ii) analytical simulation in 1D and 2D; (iii) scatter field generation; (iv) HGL examples. The paper ends with summary, discussion, and suggestions for future work.
\end{abstract}

Keywords: predictive simulation; structural health monitoring; SHM; piezoelectric wafer active sensors; PWAS; guided waves; nonreflective boundaries; WFR, NRB, hybrid global local; HGL; FEM; FEA, CMEP

\section{INTRODUCTION}

\subsection{Background and Motivation}

Structural health monitoring (SHM) sets forth to determine the health of a structure by monitoring a set of structural sensors. The SHM system registers if a damaging event has occurred, localizes and quantifies the structural damages, and monitors the damage progression over time (Figure 1). By assessing the remaining useful life (RUL), the SHM system may advise on the need for structural actions, such as repairs or load reduction. At present, SHM is seen as an complement to existing nondestructive evaluation (NDE) practices. Built-in SHM system capable of detecting and quantifying damage would increase the operational safety and reliability, reduce the number of unscheduled repairs, and bring down overall maintenance costs.

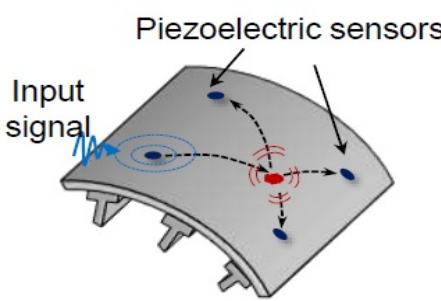

Structure with SHM system based on ultrasonic waves

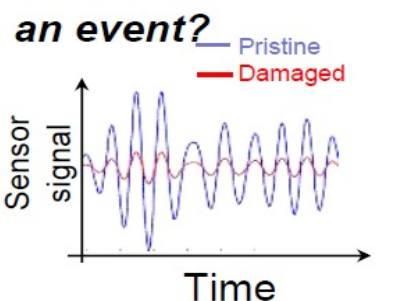

1. Detection of damage

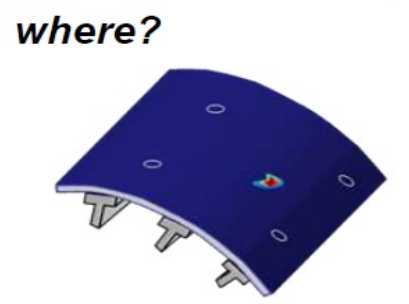

2. Localization of damage

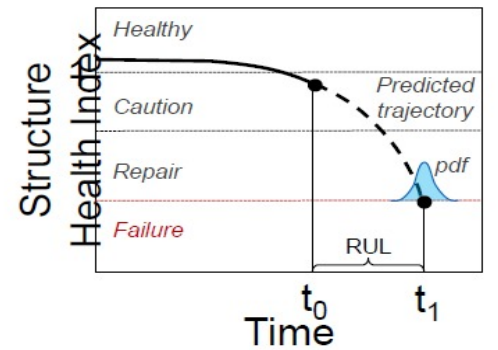

4. Prognostics \& health management

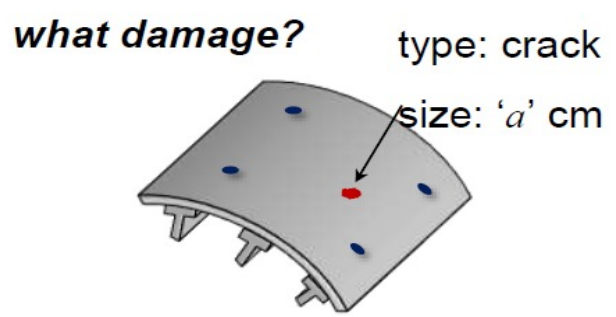

3. Classification \& quantification of damage

Figure 1: The SHM steps from damage detection to remaining useful life (RUL) estimation [1] 


\subsection{SHM Methods}

SHM methods can be either vibration based [2] [3]; or wave based [4] [5] [6]. Wave-based SHM (aka, acoustoultrasonics) works in both passive and active modes. It is able to capture the waves produced by a damaging event (passive sensor diagnosis, PSD [4]) as well as characterize the damage through diagnostic-wave interrogation (active sensor diagnosis, ASD [4]). The structural waves are generated and captured with small piezoelectric wafer transducers adhesively bonded to the structure [7] [8] [9]. We call these transducers piezoelectric wafer active sensors (PWAS) [10]. This approach has been successful in emulating the guided-wave NDE methods (pitch-catch, pulse-echo, phased array [10] [11] [12] [13] [14] [15] [16]) as well as in developing new techniques such as the electromechanical (E/M) impedance spectroscopy (EMIS) [17] [18] [19] [20].

\subsection{Multi-site Fatigue Damage; Widespread Fatigue Damage (WFD)}

Metal fatigue is a natural phenomenon in the aircraft practice. The light-weight requirements of aircraft design are such that all aircraft are subjected to metal fatigue sooner or later. Aircraft structural integrity programs based on periodic NDE inspections exist to ensure that aircraft operation is safe and reliable. Nonetheless, surprises appear: a fatigue issue that has produced several near-accidents (Aloha Airlines 1988 in-flight decompression [21], Southwest Airlines 2009 and 2011 rapid decompression [22] [23], etc.) is that of multi-site fatigue damage aka widespread fatigue damage (WFD) [24]. The WFD phenomenon appears in mechanically-fastened splice joints (Figure 2a). What happens is that cracks emanating from adjacent rivet holes are joining together to produce 'unzipping' of the splice joint (Figure 2b).

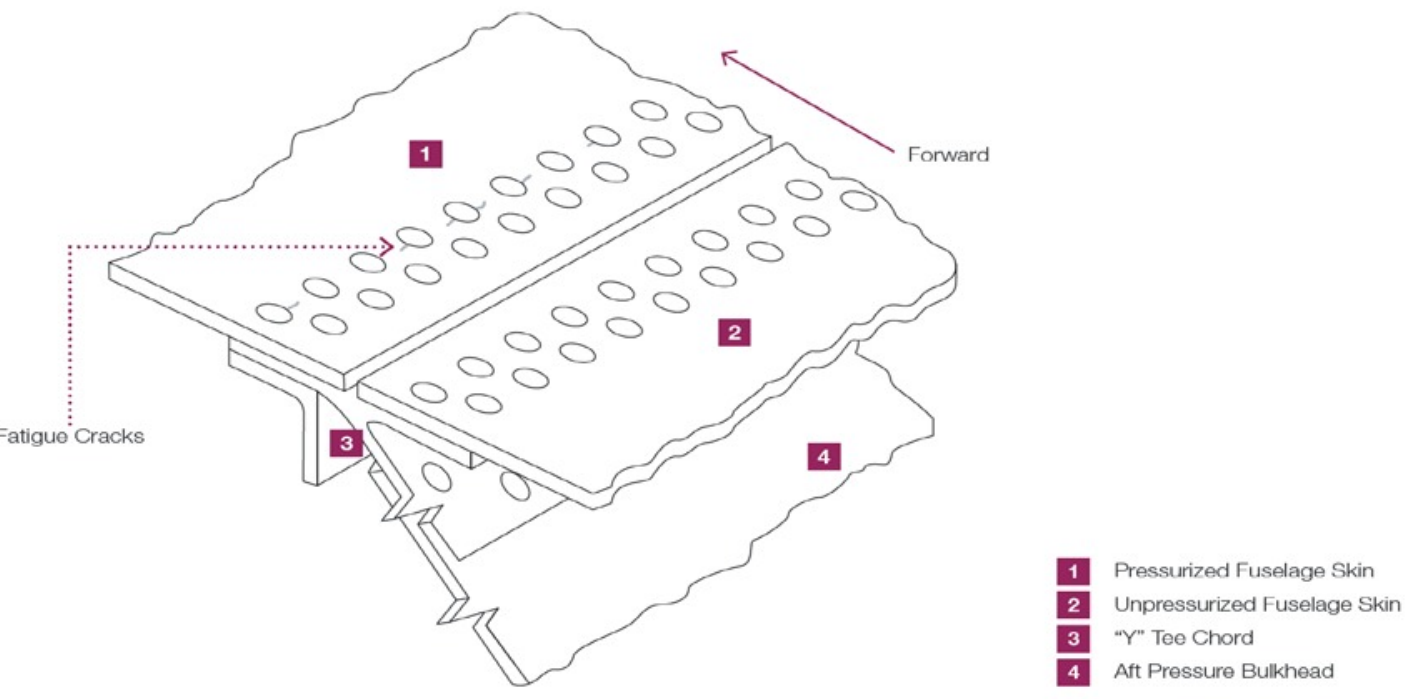

(a)

(b)

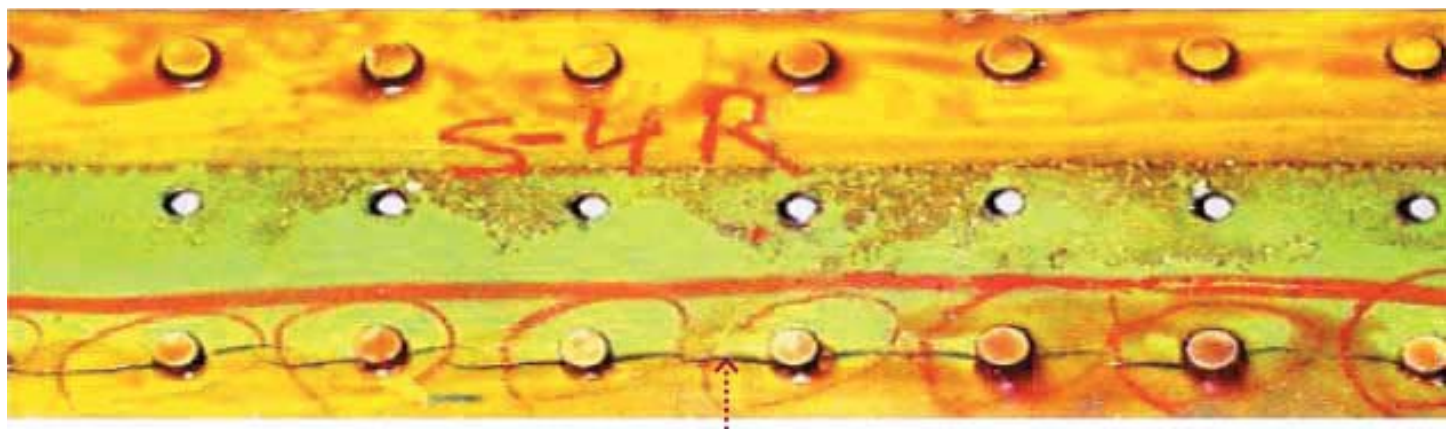

Figure 2 Widespread fatigue damage (WFD) example: (a) structural splice with multiple fastener holes and cracks prone to WFD; (b) photo of actual WFD phenomenon observed on a row of rivets in an aircraft splice joint [24] 


\subsection{Early Detection and Prevention of WFD}

The WFD phenomenon is related to butterfly cracks emanating from the fastener holes (Figure 2b). As shown in Figure 3 , a hole placed in a stress field creates stress concentration sites on its sides. These stress concentration sites facilitate the lateral growth of fatigue cracks. Due to their symmetric arrangement lateral from a rivet hole these fatigue cracks are known as 'rivet-hole butterfly cracks' (Figure 3). The rivet-hole butterfly cracks must be detected early, i.e., before they reach the size where the WFD may happen.

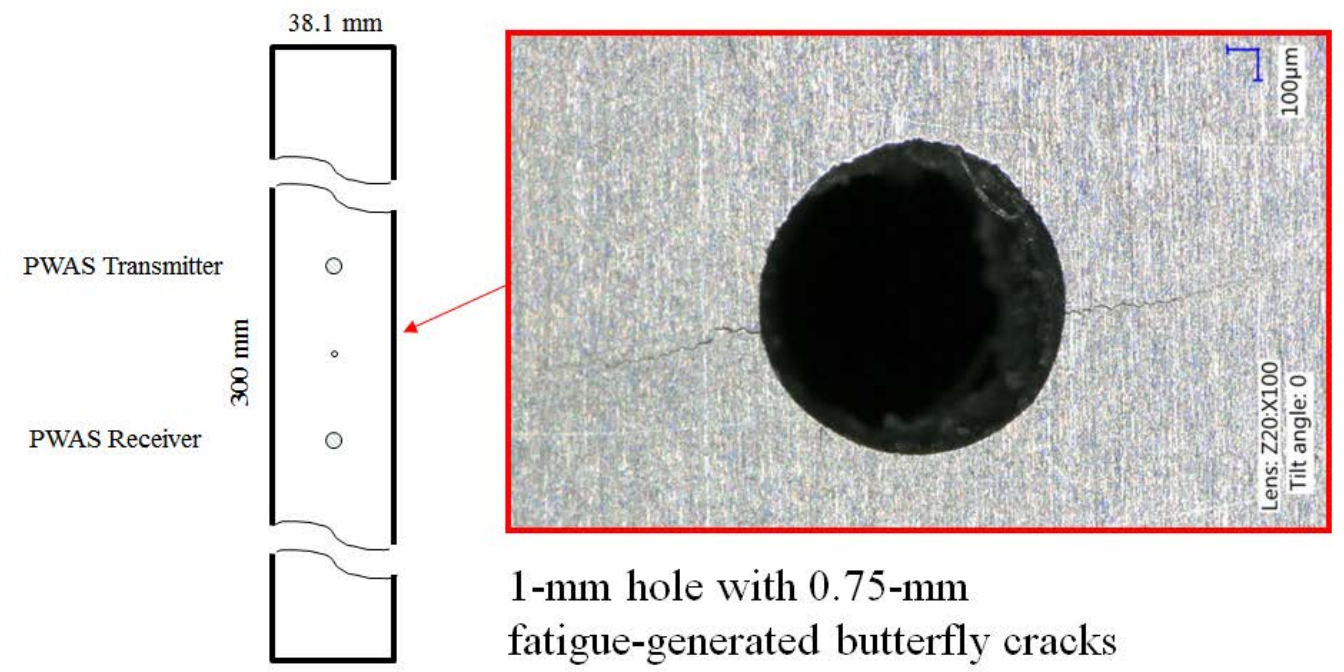

Figure 3 Butterfly cracks growing from the stress concentration on the sides of rivet hole [62].

\subsection{How could SHM Detect Rivet Hole Butterfly Cracks and Prevent WFD?}

Figure 4a presents a conceptual representation of how PWAS transmitter $(\mathrm{T})$ and receiver $(\mathrm{R})$ transducers could be used to detect the presence of rivet hole butterfly cracks and monitor their growth. The PWAS transmitter pings with a tone burst; the resulting wave packet is scattered by the rivet hole; the scattered field is detected by the PWAS receivers, including the transmitter PWAS which acts in dual mode and now has become a receiver. However, the detection of the butterfly crack is not straight forward because the received signal has multiple packets in it which may partially overlap. The packets in the received signal may be due to:

(a) scatter from the hole itself; a clean "pristine" hole is nonetheless a scatterer with its own scatter pattern. The scatter due to the butterfly crack is superposed on top of the scatter from the clean hole

(b) reflection from the splice joint edge; this reflection could be normal of oblique depending on the relative arrangement of the transmitter and receiver. Though the edge reflection is expected to arrive later than the hole scatter, it may still be difficult to separate in the signal.

One way to deal with this situation is through the differential signal method. In this case, one has at one's disposal a stored signal representing the pristine structure with no cracks. By subtracting the 'pristine' signal from the 'damage' signal, one could obtain that component of the signal which is due only to the scatter from the damage. Such an exercise is illustrated in Figure 4b which shows that the reflection from a rivet-hole crack can be separated out quite adequately under laboratory conditions.

\subsection{Variability and Uncertainty Effects in Processing SHM Data}

Although the signal differential method has promise, its practical implementation in SHM work may encounter considerable difficulties due to variability and uncertainty factors. These confounding effects may occur in design, manufacturing, maintenance, repairs, modifications, usage, etc. Since it is known that variability is stochastic in nature [25], boundary scatter/reflection effects may vary stochastically for one structure to another. Variability in what we measure and how we measure leads to uncertainty in the conclusions we draw. External factors, such as environment and operating conditions, may affect the damage sensing capability of the SHM system. Experimental studies of these effects 
have covered variation in temperature [25] [26] [27] [28] [29] [30] [31] [32] [33], operational loads [34], combinations thereof [35] [36], vibration [37], and boundary conditions [38]. Ref. [39] indicates that over 20 factors influence the SHM signal from a damage site when inspecting for fatigue cracks in multi-layered structures. Some compensation techniques have been attempted but only in simple cases [27] [32].

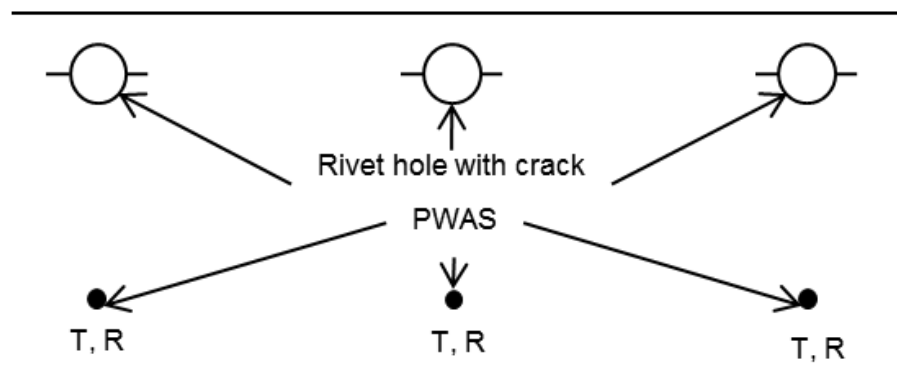

(a)

Figure 4 Conceptual representation of how PWAS transmitter $(T)$ and receiver $(R)$ transducers could be used to detect the presence of rivet-hole butterfly cracks and monitor their growth; (b) experimental results of crack detection with the signal differential approach [10], p. 894

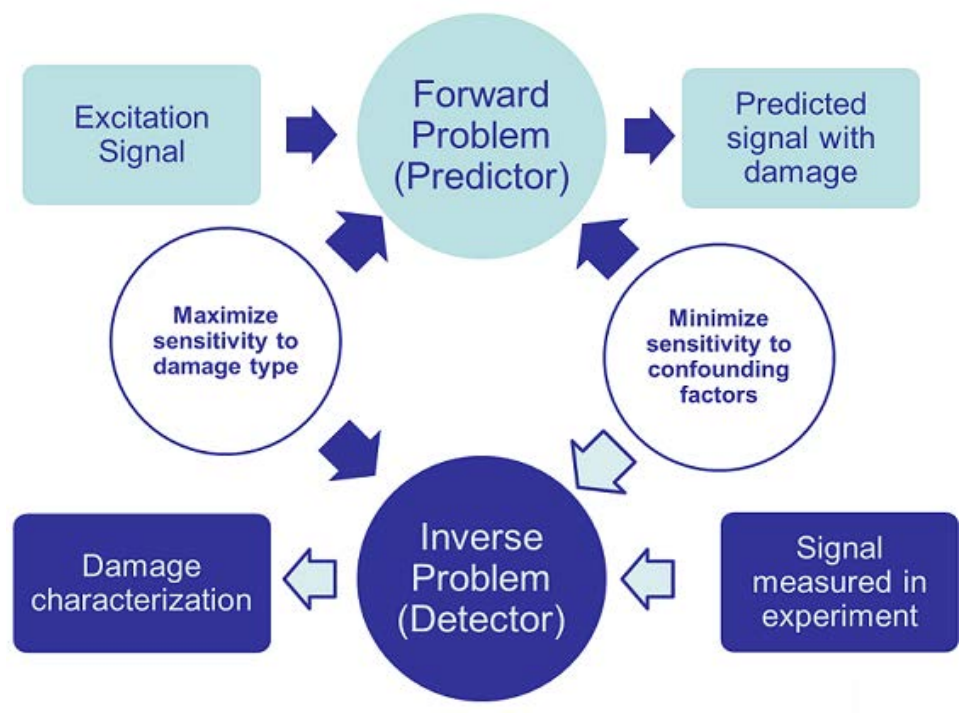

Figure 5: Model-assisted SHM-system design methodology conceptualized as predictor-detector pair

\subsection{The Robust SHM System}

A robust SHM system is needed to overcome the variability and uncertainty challenges. Such a robust SHM system must have good sensitivity to damage presence and size while being virtually insensitive to variability and uncertainty confounding effects. Of course, such a robust SHM system does not yet exist. In order to achieve it, one needs an 
appropriate SHM-system design methodology. We propose a model-assisted SHM-system design methodology as illustrated in Figure 5. Such an approach should combine numerical simulation, experimental validation, and statistical data processing. As shown in Figure 5, the SHM design process consists of two arms: the first arm is a predictor arm (forward problem), which generates the signal that that the sensors would receive for a given excitation in a given structure under given ambient and loading conditions. The second arm is a detector arm (inverse problem) which would take as input the measured SHM signals and detect if damage exist and characterize its size and location. This inverse problem is the more difficult of the two: besides being ill-posed and possibly non-unique (different factors may produce same apparent signal changes) it is also heavily influenced by the confounding factors mentioned earlier. However, the synergistic pairing of the forward problem (predictor) and inverse problem (detector) indicated in Figure 5 may yield an optimized SHM system design that maximizes sensitivity to damage while minimizing sensitivity to the confounding factors (environment, load, boundaries, etc.)

\subsection{What is it Needed?}

In order for the robust SHM system design methodology described in Figure 5 to be achieved, one has to do a large number of design iterations for various combinations of damage types and sizes, loading cases, and ambient conditions. For each case, one would have to use the forward problem to generate a set of signals which would then be fed into the inverse-problem detector to produce a damage characterization outcome. On the one hand, it is apparent that doing the forward problem through actual experiments, thought technically possible, it would require extensive resources and thus become impractical. On the other hand, the approach would be more practical if the forward problem were done in simulation using an efficient but sufficiently accurate computational approach. The search for such an efficient and faithful predictor capable to capture the damage details with sufficient accuracy while being computationally rapid makes the object of the hybrid local approach (HGL) discussed in this paper.

\subsection{The Layout of this Paper}

The paper starts with a short review of the hybrid global local (HGL) approach. Then, the paper presents the methodology for analytical guided wave simulation in the global field in interaction with PWAS transmitters, PWAS receivers, and scatterers. Both 1D (straight crested) and 2D (circular crested) guided wave propagation situations are considered. This methodology includes multi-wave-mode interaction and mode-wave-tuning for finite-size PWAS transducers as well as PWAS impedance concepts that allow the analytical simulation of mechanical wave response as a result of an electrical input applied to the PWAS transmitter and, vice-versa, the electrical signal generated by a PWAS receiver in response to an incoming mechanical wave.

The paper continues with a presentation of the methodology for predicting the scatter field generated from the interaction between guided waves and damage. The concept of local FEM high-density high-accuracy meshing is presented. The framework for extracting the wave-damage interaction coefficients (WDIC) is presented for the fundamental A0, S0, SH0 modes. The local FEM region is extended to partially overlap with the global analytical region such that the scatter from an incoming guided wave can be captured and identified with the appropriate A0, S0, SH0 modes. Nonreflective boundaries (NRB) are used to prevent boundary reflections from the edges of the finite size FEM region.

The last part of the paper discusses examples such as the scatter of guided waves interacting with a crack in a 1D waveguide and the scatter from a rivet-hole butterfly crack in a 2D plate. It is shown that certain frequency-wave mode combinations may facilitate detection whereas others combinations may actually impede detection.

\section{HYBRID GLOBAL LOCAL (HGL) ANALYSIS}

The hybrid global local (HGL) concept can be traced back to Goetschel et al. [40] that used with different FEM mesh sizes and variable granularity to simulate the elastic bulk wave propagation in the global domain and the interaction of waves with scatterers in the local domain. Further developments of the bulk-waves HGL approach [41] [42] have culminated with the full-scale CIVA simulation package that has been used extensively in the nuclear industry [43] [44]. For NDE and SHM of thin-wall structures, as specific to aerospace applications, the guided-wave approach offer specific benefits over the bulk-wave approach. However, the multi-modal character of the guided waves makes such problems an order of magnitude more difficult than the bulk-wave problems. To address guided-wave NDE of aerospace structures, Mal and co-workers envisioned in the late 1990s [45][46][47] a combination of closed-form analytical solution in the global domain and FEM solution in the local domain to achieve an efficient simulation of guided wave propagation and interaction with damage in thin plates. Exact displacement continuity and traction balance were imposed at the boundary 
between the local FEM and global analytical domain through colocation or an equivalent approach. An HGL application to arbitrary waveguides using the semi analytical finite element (SAFE) method is described in ref. [48].

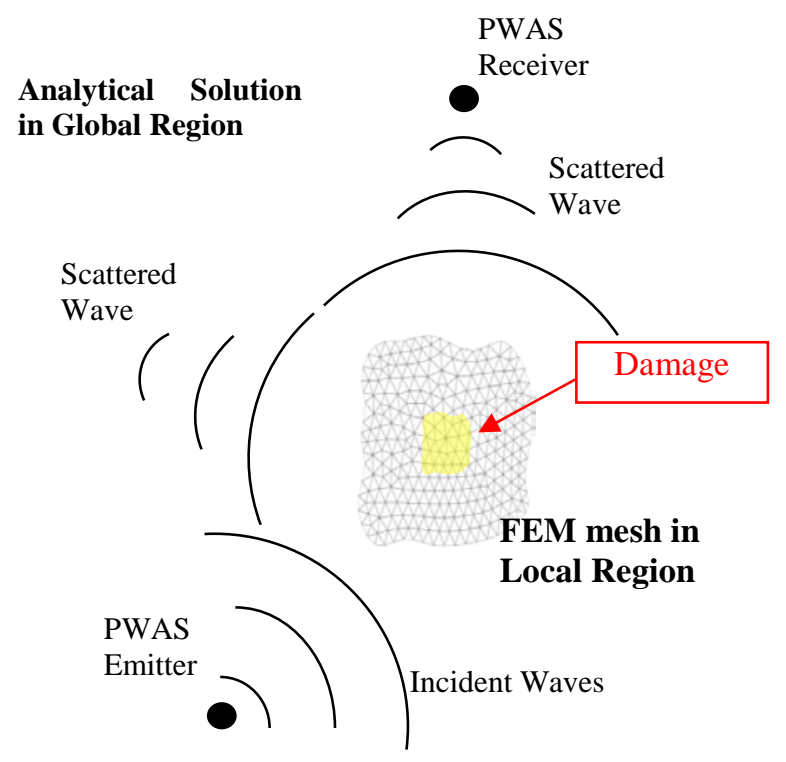

Figure 6: General 2D set-up for Hybrid Global-Local modeling of structural sensing.

Our LAMSS group extended the HGL approach to SHM applications using PWAS transducers. We were inspired by the 1990s seminal work of Chang and Mal [45] [46], but we had to substantially modify it in order to avoid the solution of the large over-determined set of complex equations at the global-local interface. In contrast with ref. [45] [46], we adopted a different approach that combines frequency-domain and time-domain solutions and uses a transition overlap between the local and global regions.

Our HGL methodology incorporates an analytical framework for guided wave propagation in the global region (called Wave Form Revealer or WFR) and a local FEM analysis with nonreflective boundaries (NRB) for the determination of the wave damage interaction coefficients (WDIC) [49] [51] [52].

In our approach, the guided waves are generated with a PWAS transmitter, scatter from a damage, and get picked up by a PWAS receiver (Figure 6). The travel from transmitter PWAS to the damage and from the damage to the receiver PWAS is modeled with analytical wave propagation formulae, whereas the interaction between the guided waves and the damage is done with FEM discretization [49]. As different from ref. [45] [47], the LAMSS approach to the HGL method is to replace the damage with a new guided wave source that generates the scatter field to be added to the analytical solution. The scatter field is defined in terms of wave damage interaction coefficients (WDIC) that are calculate through a local FEM analysis or by other methods.

\section{ANALYTICAL GUIDED WAVE SIMULATION FOR STRAIGHT CRESTED AND CIRCULAR CRESTED GUIDED WAVES}

The first step taken in our HGL approach was to build a simulation environment that allows for a fast and efficient modeling of the guided waves generation by a PWAS transmitter, their propagation throughout the thin-wall structure, and then their sensing by the PWAS receiver. This simulation environment and the associate graphical user interface (GUI) has come to be know as WaveForm Revealer (WFR) [50] [51].

Though the guided waves are elastodynamic phenomena, their generation is done under electric excitation through the converse piezoelectric effect in the PWAS transmitter, whereas their sensing in the PWAS receiver is done in the form of electric signals obtained through the direct piezoelectric effect in the PWAS transducer. Thus, the problem has a multiphysics character. In addition, the finite size of the PWAS transducers produces tuning effects, i.e., at various frequencies, various guided wave modes may be excited or sensed differently depending on the relative ratio between their wavelength and the PWAS size (see ref. [10], Chapter 11). Besides guided wave generation, propagation, and 
reception, the WFR simulation environment allows for insertion of damage scatterers at user-defined locations through the use of complex-numbered WDIC values.

Two WFR GUIs have been developed, one for straight-crested guided waves (WFR-1D), the other for circular-crested guided waves (WFR-2D).

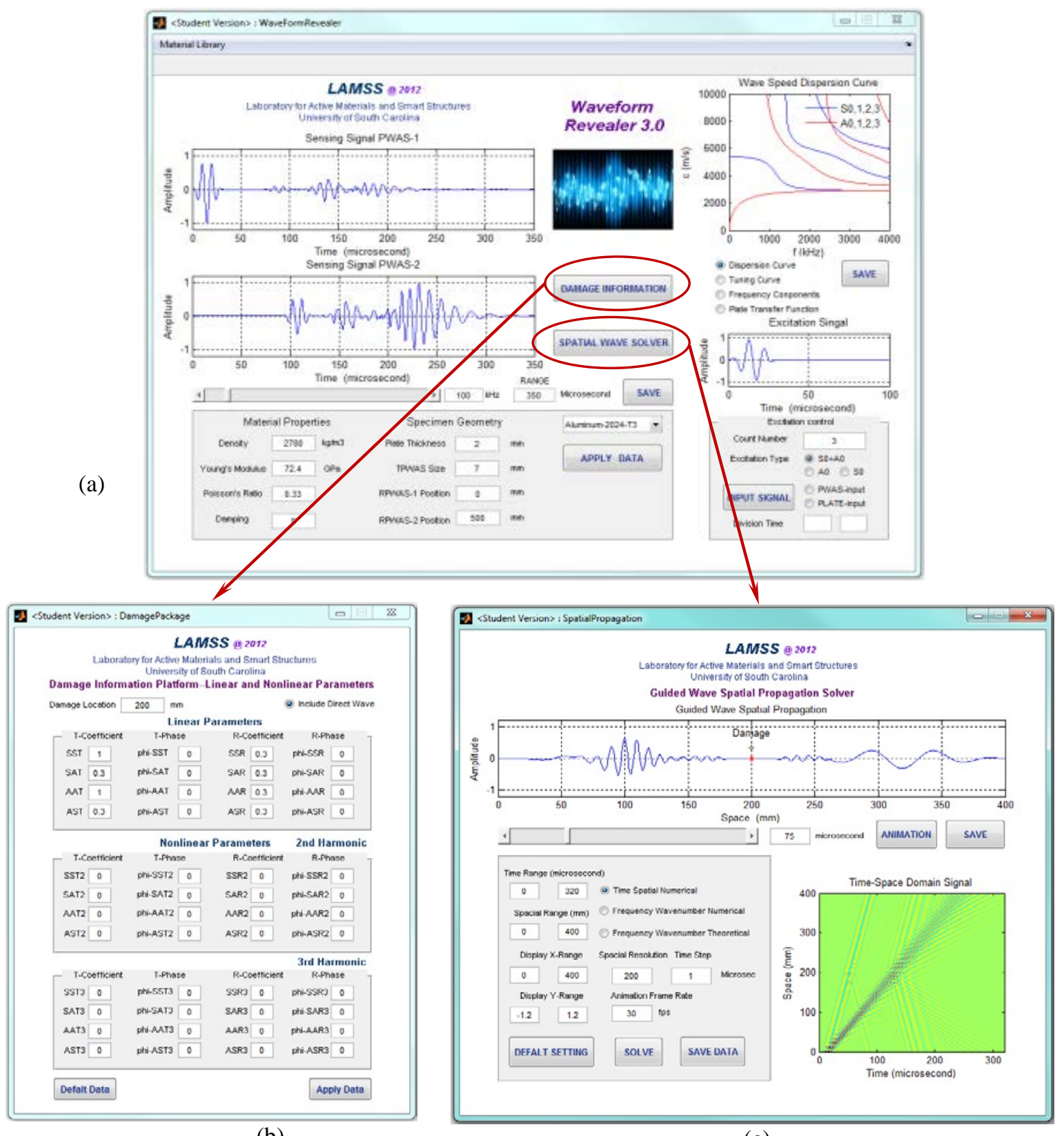

(b)

(c)

Figure 7: (a) WFR-1D GUI; (b) damage information platform; (c) guided wave spatial propagation solver

\subsection{WFR-1D}

The WFR-1D assumes straight-crested guided waves, hence the problem is $y$-invariant and the wave propagation is 1D. The closed-form analytical solution of this multi-physics problem [51][53] was coded in MATLAB. The resulting GUI WFR-1D is shown in Figure 7a. The spatial propagation solver is like a B-scan. Using the analytical procedure, we obtain the time domain waveform solution at various locations in the structure. Thus, the time domain waveform 
solutions of a sequence of points along the wave propagation path are obtained. If we select the sequence of solution points fine enough, a time-spatial domain solution of the wave field is obtained. The spatial solution of wave field at a particular instance in time is available as shown in Figure 7b. After the time-space solution of the wave field is obtained, one can also do the frequency-wavenumber analysis to see the wave components of the signal. These will be illustrated in the case studies discussed later in this paper.

\subsection{WFR-2D}

The WFR-2D assumes circular-crested guided waves, hence the solution involves Bessel and Hankel functions [53]. Figure 8a shows the WFR-2D main interface which calculates in real time the sensing signals as well as the dispersion curves and tuning curves. The parameter control panel allows users to modify the material properties, structural thickness, and transmitter-damage-sensing locations. The excitation control panel provides excitation waveform, frequency, and arbitrary excitation loading options. Users can also selectively choose the excited wave mode of interest. Figure 8b shows the damage information platform for inputting wave-damage interaction coefficients (WDICs). Figure 8c and Figure 8d show the sub-interfaces for loading S0 and A0 WDICs. The PWAS properties module allows users to define PWAS geometric and material properties (Figure 8e). The spatial propagation solver (Figure 8f) calculates the transient time-space domain wave field and produces a wavefield image that can identify the damage location like a conventional ultrasonic C-scan.

(a) WFR Main Interface

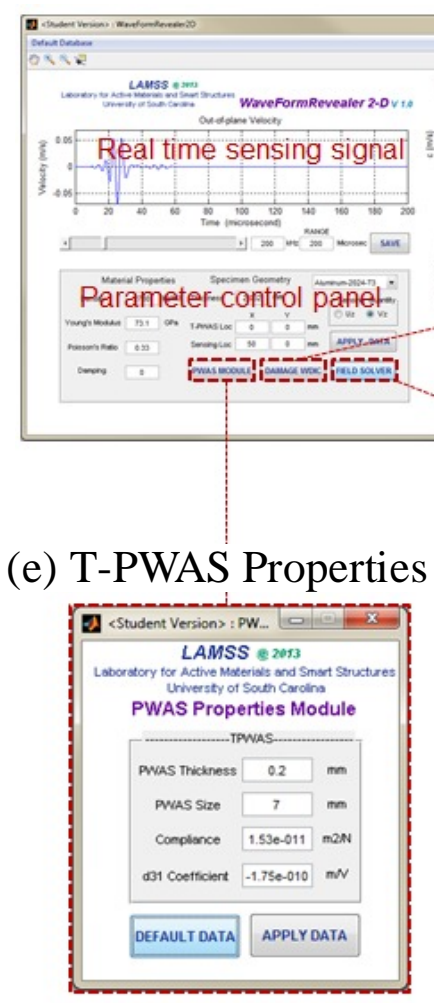

(b) Scatter Information Platform

(c) S0 WDICs Module

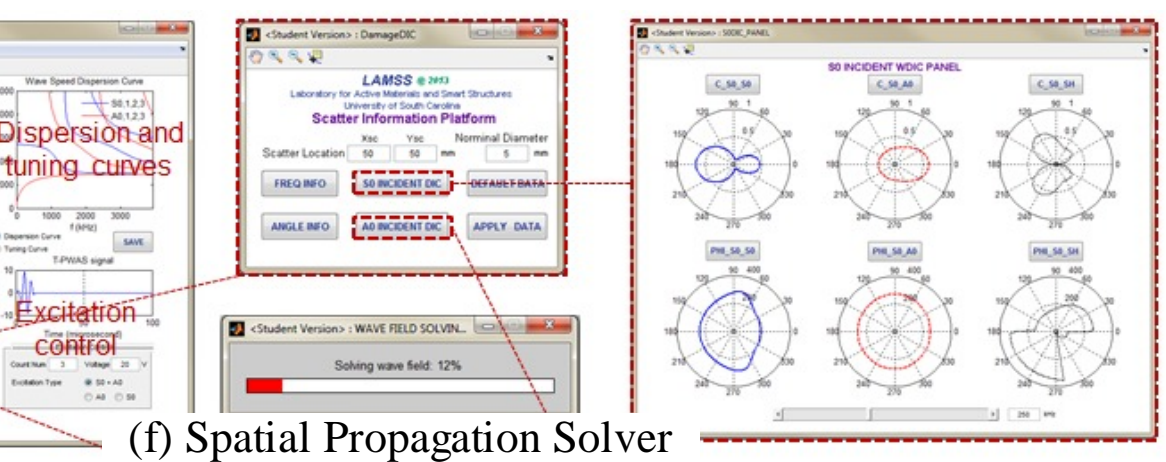

(f) Spatial Propagation Solver

(d) A0 WDICs Module

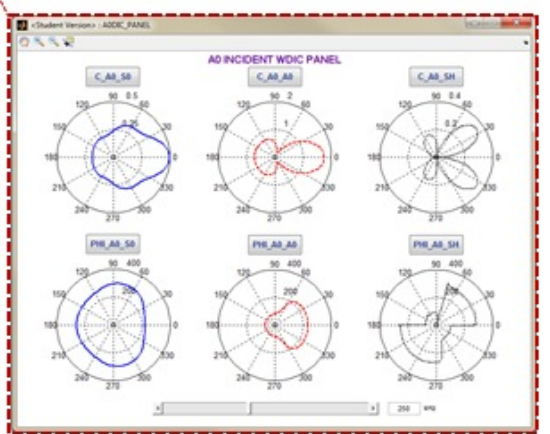

Figure 8: WFR-2D GUI: (a) WFR-2D main interface; (b) damage information platform; (c) So WDICs module; (d) A0 WDICs module; (e) T-PWAS properties module; (f) spatial propagation solver.

\section{SCATTER FIELD SIMULATION}

Two methods for predicting the scatter field generated by the interaction of guided waves and structural damage were explored. The first method consisted of a local FEM analysis in which nonreflective boundaries (NRB) are placed on the 
extremities of the FEM mesh. Thus, the analysis can be performed as the damage inclusion was part of an infinite domain without unwanted reflections from the boundaries. This ensures that no standing waves are created and a pure scatter phenomenon is simulated. The second method consisted in developing an exact analytical solution of the scatter phenomenon using a series expansion onto all the propagating and nonpropagating guided wave modes present in the structure in the vicinity of the damage. Thus, the scatter field is generated analytically through a rapidly convergent series expansion that can be truncated to the desired accuracy.

(a)

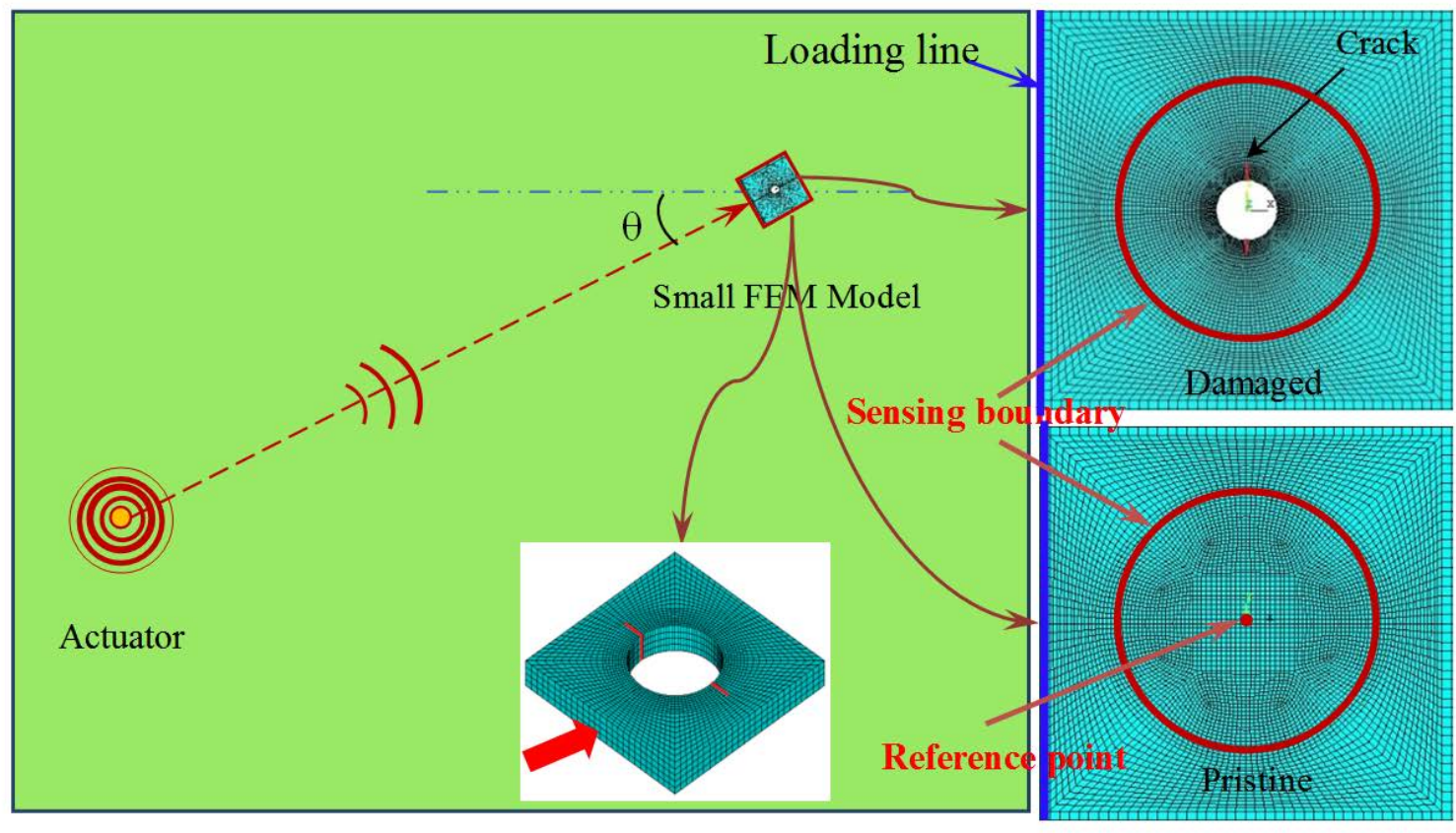

(b)
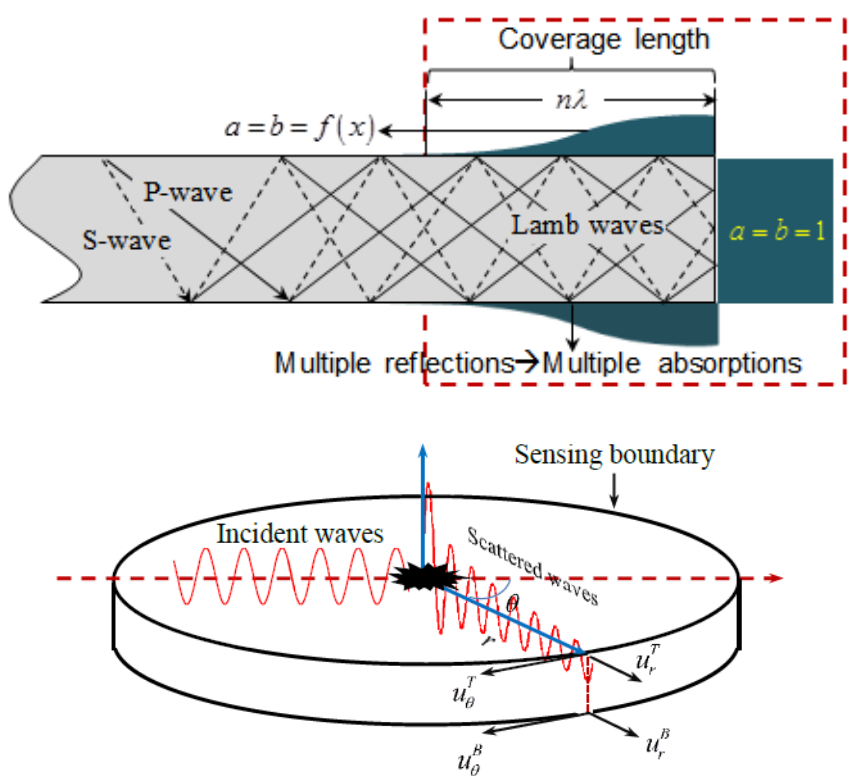

Figure 9: FEM analysis to determine wave damage interaction coefficients (WDIC) values: (a) overall view; (b) nonreflective boundary details; (c) extraction WDIC values 


\subsection{FEM with Nonreflective Boundaries}

Shen and Giurgiutiu [52] developed an NRB approach that effectively absorbs the Lamb waves at plate free edges. This concept takes into account the fact that Lamb waves result from the superposition of $\mathrm{P}$ and $\mathrm{S}$ waves that undergo multiple reflections at the top and bottom surfaces of the plate as well at the plate end (Figure 9). Hence, the NRB must inhibit both the end reflections at the plate boundary as well the top and bottom reflections in its near vicinity. In order to achieve this, viscous boundaries were added both at the plate end and on the top and bottom surfaces near the plate end; the latter viscous boundaries were smoothed out by adopting a gradually decreasing viscosity parameter from the plate end towards the inner region. The NRB FEM analysis is done in the frequency domain such that the WDICs are generated over a wide frequency band as needed to perform the convolution with the interrogative signal generated by the transmitter PWAS.

\subsection{Analytical Scatter Field Generation with the CMEP Method}

The real, imaginary, and complex roots (wavenumbers) of the Rayleigh-Lamb equation for symmetric and antisymmetric Lamb wave modes were extracted using an efficient complex root search algorithm [54]. Thus the wave field in the vicinity of the damage was expressed as a series-expansion superposition of propagating Lamb waves (real and complex wavenumbers) and evanescent Lamb waves (imaginary wavenumbers). This ensures proper representation of the wavedamage interaction phenomena, although only the non-attenuated propagating Lamb waves (real wavenumbers) would arrive at the receiver PWAS.

The participation factors of each of these complex waves were determined from a Galerkin solution of the boundary condition differential equations at the damage discontinuity. Fast convergence of the Galerkin approach was ensured by an appropriate vector projection which mimics the power flow expressions, i.e., the traction boundary conditions were projected onto the displacement field whereas the displacement conditions were projected onto the stress field. This approach was called complex mode expansion with vector projection (CMEP) [54] [55]. The CMEP method has been successfully applied to 1D problems such as scattering from notches and vertical cracks [56] as well as fro horizontal cracks and disbonds [57]. The analytical generation of the scatter field WDIC was found to be orders of magnitude faster that the corresponding NRB FEM approach [54].

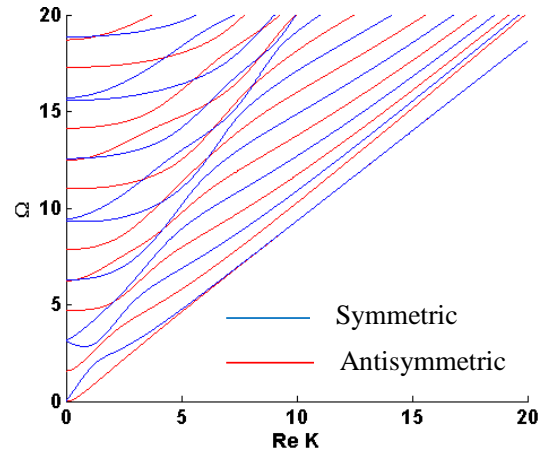

(a)

(b)

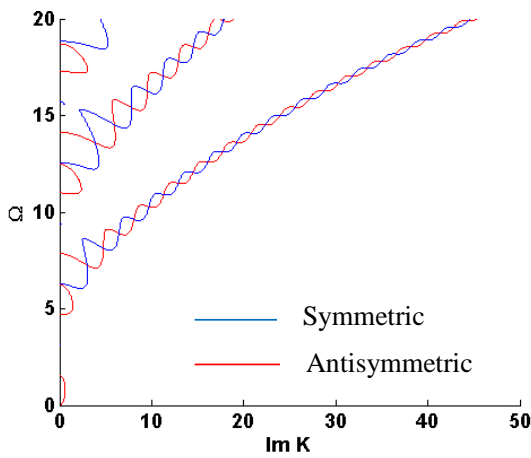

(c)

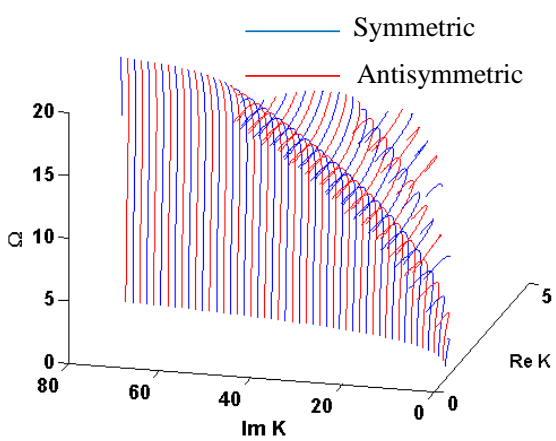

Figure 10: (a) real, (b) imaginary, and (c) complex roots of the Rayleigh-Lamb equation for $v=0.33$

\section{HGL EXAMPLES}

Examples of how the HGL method can be applied to 1D and 2D problems are given next. The 1D problem considers the interaction between a straight crested wave and a notch parallel to the wavefront. A vertical crack may be simulated by a notch of vanishing width. The 2D problem considers the interaction between a circular wave front and a rivet hole experiencing a butterfly fatigue crack. Though the rivet hole itself is a wave scatterer, the presence of the butterfly crack adds additional scattering effects. By subtracting the scatter field of the hole from the scatter field of the hole with the butterfly crack, one obtains the scatter field attributable only to the butterfly crack. 


\subsection{D Analysis of a Notch}

Figure 11 shows a comparison between simulated and experimental signals resulting from a notch scatterer. The notch with a height $h_{1}=2.5 \mathrm{~mm}$ and a width $d_{1}=0.25 \mathrm{~mm}$ was machined as a long straight grove into a 3.17-mm thick 7075T6 aluminum plate. A transmitter T-PWAS transducer was placed at $143.5 \mathrm{~mm}$ in front of the notch. A receiver RPWAS transducer is placed on the other side of the notch. The wave propagation path between the T-PWAS and the RPWAS is $303 \mathrm{~mm}$. The T-PWAS is excited with a 3-count Hanning-windowed tone burst of center frequency varying from $50 \mathrm{kHz}$ to $300 \mathrm{kHz}$ [51]. The T-PWAS placed on top of the plate produces both S0 and A0 wave packets traveling with different group velocities. As these wave packets encounter the notch, transmission, reflection, and mode conversion took place. Two types of experiments were performed: (a) pulse-echo; (b) pitch catch. In the pulse-echo experiment, the T-PWAS transducer acts as both transmitter and receiver. In the pitch-catch case, the T-PWAS acts as transmitter whereas the R-PWAS acts as receiver.

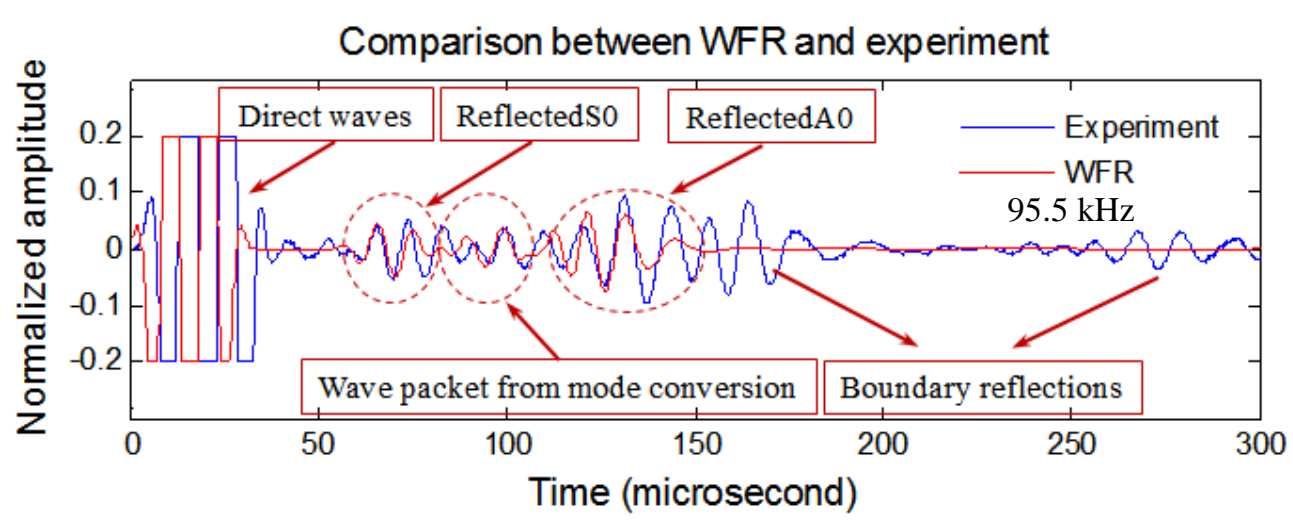

(a)

(b)

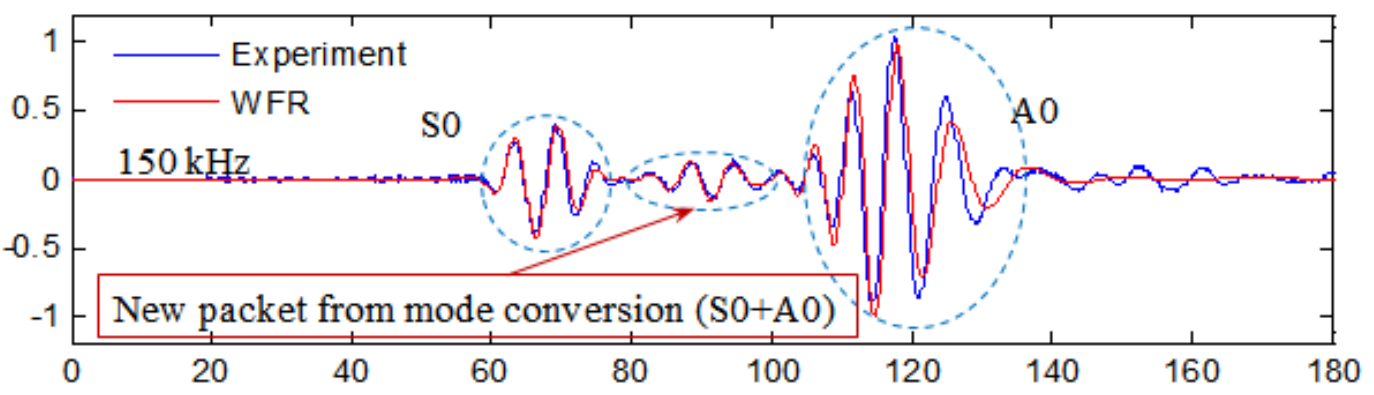

Figure 11: Comparison between WFR simulations and experiments for Lamb waves interaction with a notch: (a) pulseecho example at $95.5 \mathrm{kHz}$; (b) pitch-catch example at $150 \mathrm{kHz}$

Figure 11a shows a pulse-echo example performed with a $95.5 \mathrm{kHz}$ excitation signal. The waveform shown in Figure 11a depicts first the excitation wave (direct wave) which has a large amplitude and hence has its upper and lower peaks chopped off. The other wave packets shown in Figure 11a are the reflected S0 packet, the reflected A0 packet, and the boundary reflections. The comparison between the WFR HGL simulation and experiment reveals that the agreement with experiment is slightly better for the S0 wave than for the A0 wave. In addition, one of the boundary reflection packets superposes over the tail of the reflected A0 packet thus impeding the direct comparison with WFR predictions.

Figure 11b depicts a pitch-catch example performed at $150 \mathrm{kHz}$. In this case, the agreement between WFR HGL simulation and experiments is easier to establish because the interference from the boundary reflections is less acute. Examination of Figure 11b reveals that the S0 wave packet is predicted almost perfectly whereas the A0 wave packed has some phase differences which may be caused by a slightly different wavespeed, possibly due to variation in the plate thickness within manufacturing tolerances. The tail of the experimental A0 wave package shows some additional wave activity which may be due boundary reflections. 


\subsection{D Analysis of a Rivet Hole with Butterfly Crack}

The analysis of the scatter from a rivet-hole butterfly crack was performed in steps. For each of the S0 and A0 modes, the analysis was first performed for only a rivet hole and then for a rivet hole with butterfly cracks. For each case, i.e., pristine and damaged, the complex scatter coefficients (WDIC) are determined at a number of frequencies of interest. Next, the "pristine" WDICs were subtracted from the "damaged" WDICs to get the WDICs specific to the damage alone (here, the damage is the butterfly crack). For illustration, Figure 12 present a few results for the case of an incident S0 Lamb wave at a number of frequencies up to $1000 \mathrm{kHz}$.

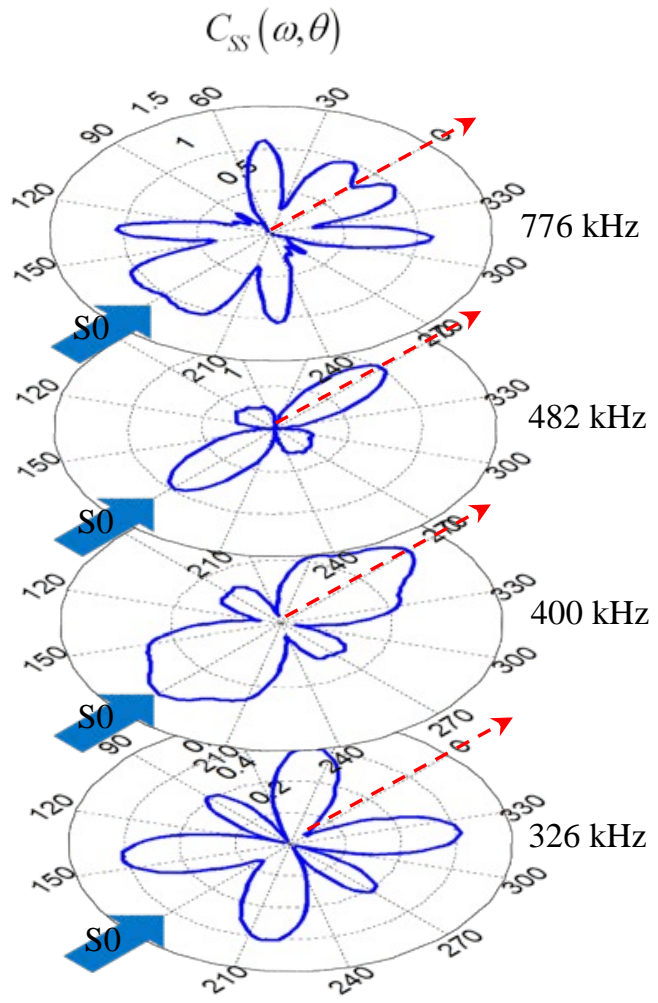

(a)
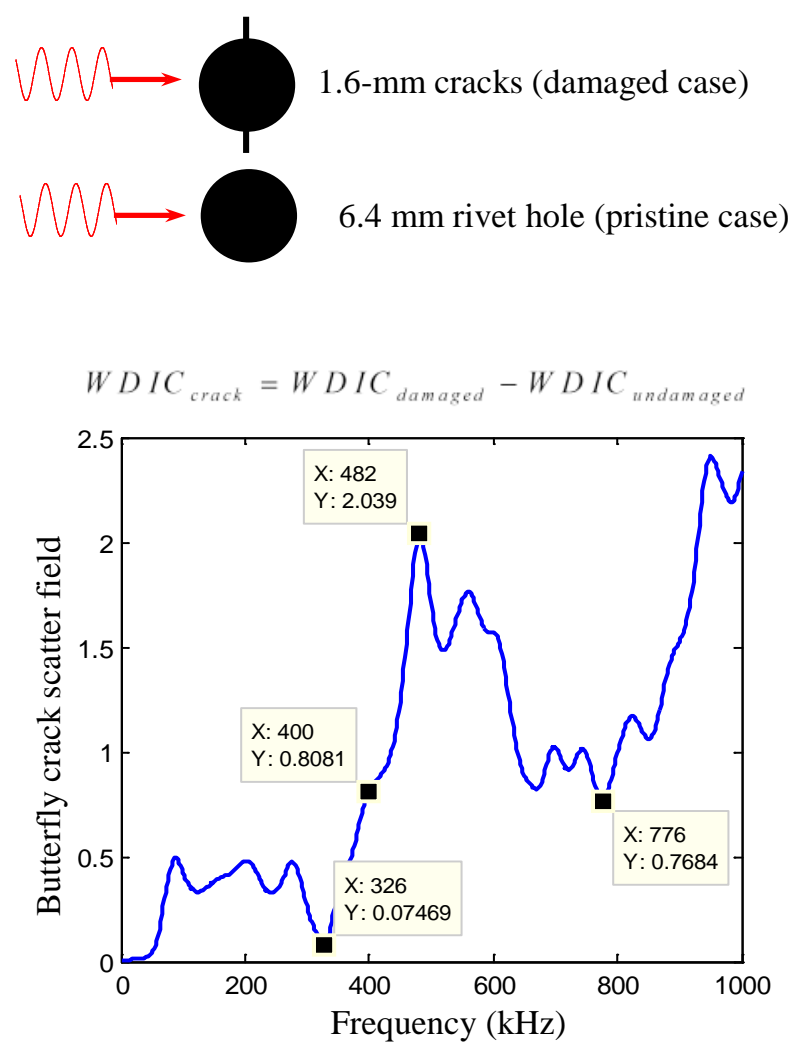

(b)

Figure 12: (a) WDIC directivity plots under various frequencies; (b) WDIC in the forward direction as a function of frequency.

Figure 12a shows the polar plot of the $C_{S S}(\omega, \theta)$ amplitude which is the WDIC for the S0-wave component of the scatter field from an incident S0 wave. It can be observed that the scattered wave field varies azimuthally with the angle $\theta$ creating specific directivity in the polar plot. These polar plots vary dramatically with the frequency $\omega$. In some cases, the forward and backward scatter lobes seem to be the strongest. (The forward direction, which is perpendicular onto the butterfly crack, is indicated by arrows in Figure 12a.) However, there are frequency values for which the forward direction seems not to be able to sense the scatter field, e.g., the $326 \mathrm{kHz}$ frequency shown at the bottom of Figure 12a.

When plotting the forward scatter field vs. frequency, we observe peaks and valleys as indicated in Figure $12 \mathrm{~b}$. Examination of this plot indicates that an almost zero is achieved at $326 \mathrm{kHz}$, whereas a local maximum seems to appear at $482 \mathrm{kHz}$. Similar results were obtained for the A0 incidence, but they will not be presented here for sake of brevity. For the same reason, mode conversion effects are also not presented. 
Using this efficient approach, we studied the butterfly-crack problem (Figure 13a) and performed optimization studies to identify the optimum SHM setup. We found that certain interrogation frequencies strengthen the scatter from the butterfly-crack (e.g., $\sim 482 \mathrm{kHz}$ in Figure 13b) whereas others would have an opposite effect (e.g., $~ 330 \mathrm{kHz}$ in Figure 13b). The optimal $482 \mathrm{kHz}$ frequency gives very clear signals (Figure 13c). An experimental confirmation of this approach was presented in [10], p. 894 (Figure 13d).

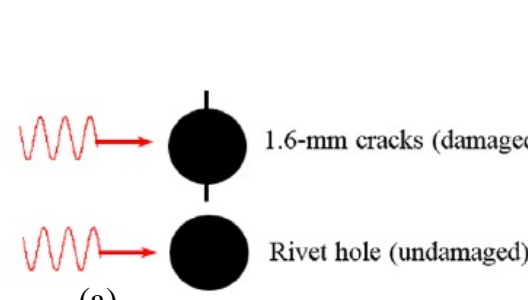

(a)
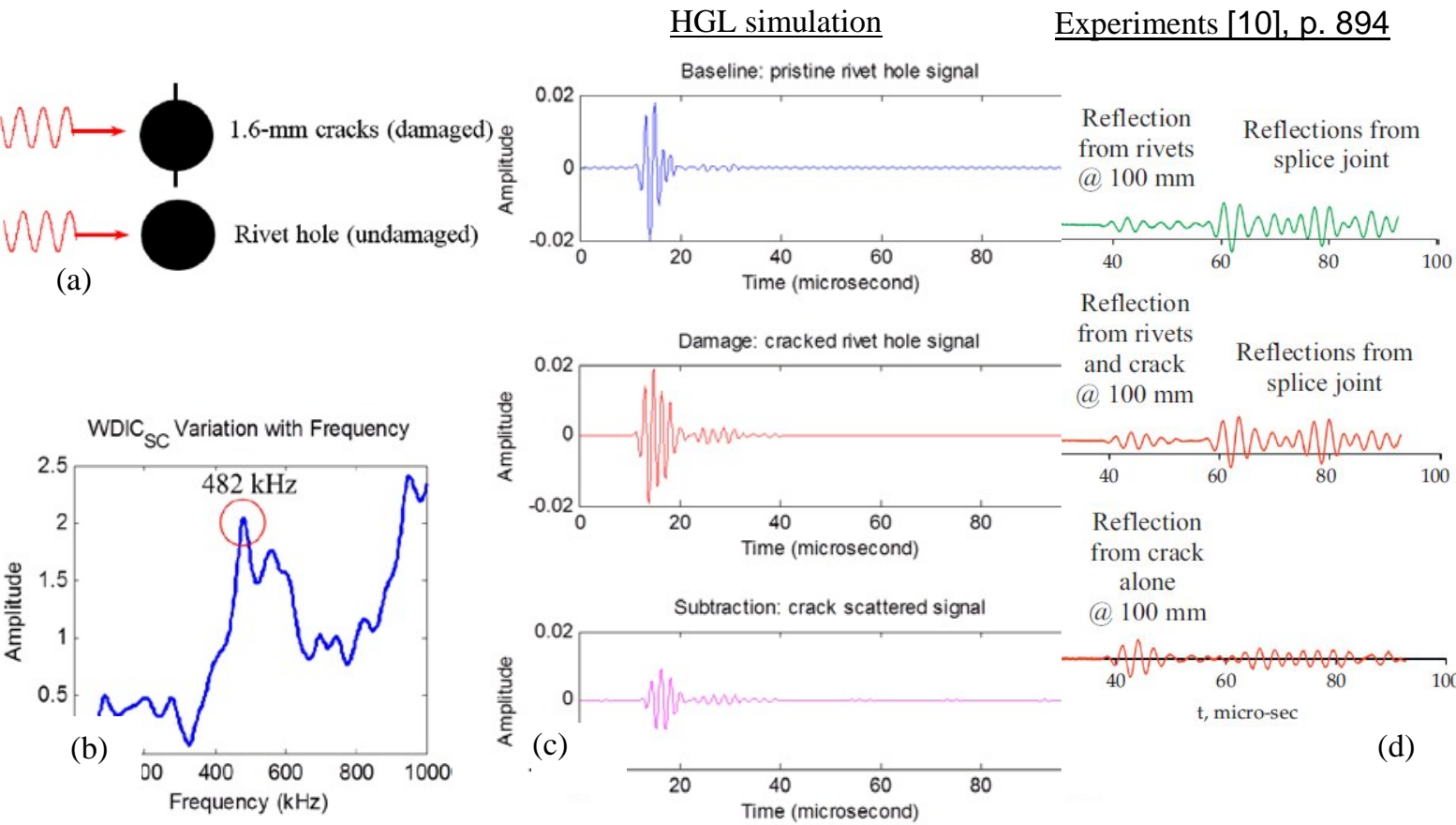

Reflection
from rivets

and crack Reflections from (a) $100 \mathrm{~mm} \quad$ splice joint

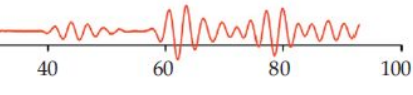

Reflection

from crack alone (a) $100 \mathrm{~mm}$

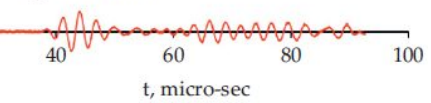

(d)

Figure 13 SHM signals from a damaged fastener hole (butterfly crack): (a) damaged vs. pristine hole; (b) optimum SHM interrogation frequency for detecting the butterfly crack; (c) sequence of simulated scatter signals [50] yielding the crack-detection signal; (d) experimental confirmation [10], p. 894

Since, the additional scatter field due only to the butterfly crack damage is frequency dependent, the possibility of an optimal interrogation frequency arises. Figure 13c shows how the large scatter field amplitude observed at $482 \mathrm{kHz}$ could be instrumental in constructing an SHM interrogation system that can easily detect the butterfly crack. As shown in the bottom right plot of Figure 13c, the wave packet due to the butterfly crack alone is very strong indeed. Also noticed in Figure 12b is the fact that certain frequencies might be inappropriate for SHM use because the scatter field would be very small, e.g., the $326 \mathrm{kHz}$ frequency for which the scatter field amplitude is almost zero. However, for other damage types, or for other butterfly crack orientations and interrogating wave modes, the best detection frequency may vary and other preferred frequencies may appear.

Rivet hole with butterfly cracks

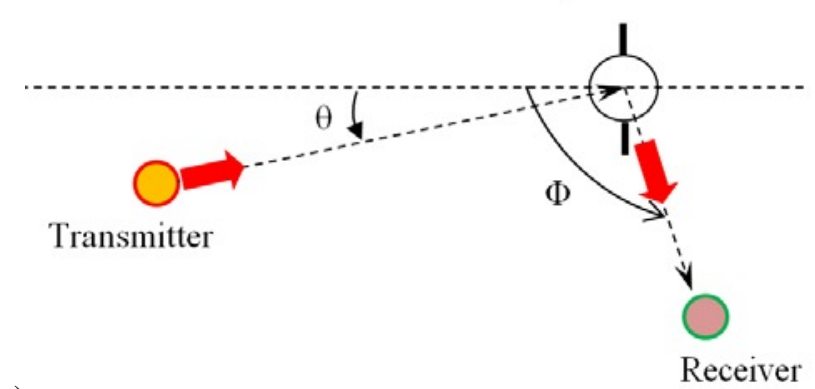

(a)

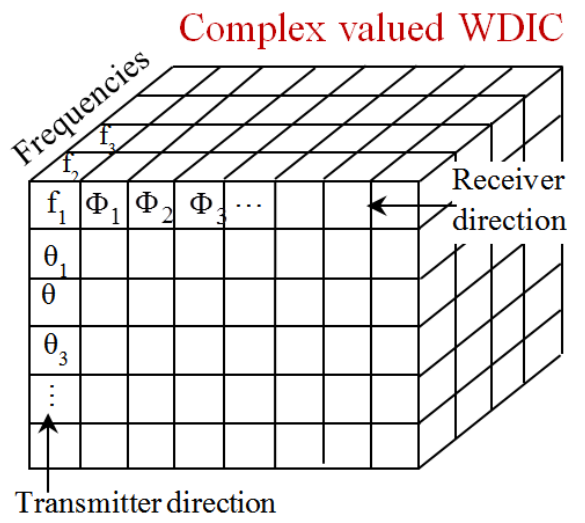

Figure 14: Transmitter and receiver directions wrt rivet hole butterfly crack; (b) WDIC scatter cube 


\subsection{Transmitter and Receiver Angle Effects in Scatter Coefficient}

The next step in our study was to investigate the effect of transmitter and receiver directions wrt to the rivet-hole butterfly crack [58]. It is apparent that, if the transmitter is not positioned on the symmetry axis of the butterfly crack, then a skew scatter pattern would arise. In addition, the position of the receiver may also vary depending on installation details. Figure 14a illustrates this situation with $\theta$ being the transmitter angle and $\Phi$ the receiver angle. Bearing in mind that one is seeking an optimal position for transducer installation, one has to explore several possible combinations of angles at different frequencies in order to find that combination $(\theta, \Phi, f)$ that is most advantageous for the detection of the butterfly crack. Thus, one has to explore a complete 'WDIC scatter cube', as illustrated in Figure 14b.

One example of skew angle incidence is illustrated in Figure 15: An S0 Lamb wave is impinging onto the cracked rivet hole at an incident angle $\theta=18^{\circ}$ measured with respect to the axis of symmetry of the butterfly crack present in rivet hole. (The axis of symmetry would be normal to the rivet row illustrated in Figure 2b and Figure 4a.) The excitation frequency is $500 \mathrm{kHz}$. The polar scatter plots presented in Figure 15 correspond to three cases: (a) the scatter field of a pristine hole showing symmetry with respect to the incident axis, as expected; (b) the scatter field of the rivet-hole with butterfly crack showing an asymmetric pattern due to the fact that the incident beam is skew wrt crack axis; (c) the scatter field of the crack-only case which was obtained by subtracting field (a) from field (b). This scatter field, which is due only to the butterfly crack, shows interesting lobes: one strong forward lobe at around $190^{\circ}$, one strong backward lobe at around $354^{\circ}$, and a side lobe around $90^{\circ}$. Similar behavior was also observed with A0 incident Lamb waves, but at different frequencies. The knowledge about the crack-only scatter lobes could be exploited when designing the SHM system to achieve an efficient placement of transmitter and receiver PWAS transducers such that they are sensitive to detecting the butterfly crack.

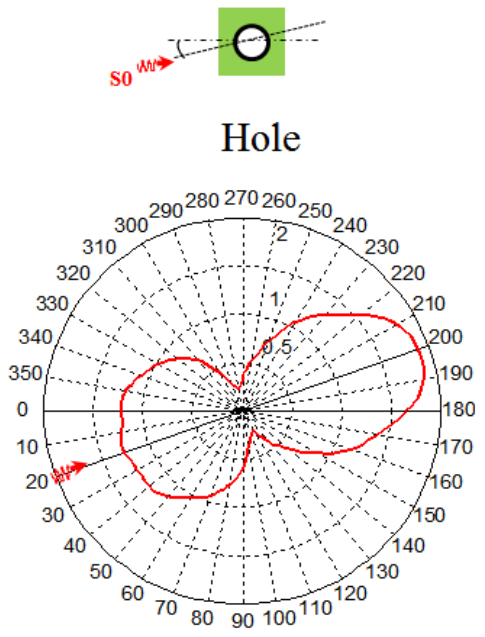

(a) pristine hole

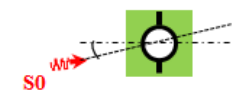

Hole+Crack

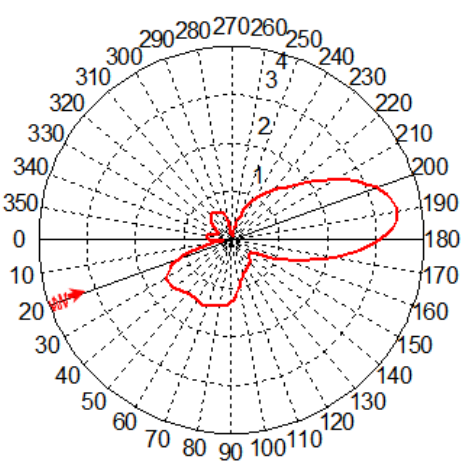

(b) cracked hole
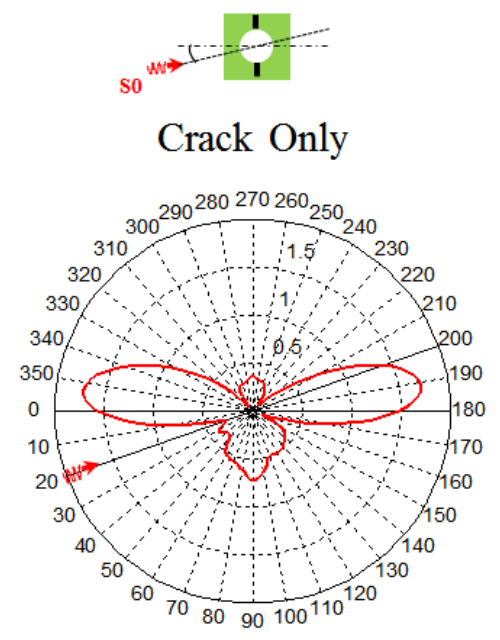

(c) crack effect only

Figure 15: The variation of the WDIC profiles under different damage situations ( $S 0$ Lamb mode incident at $\theta=18^{\circ}$, $f=500 \mathrm{kHz}, 6.4$ diameter rivet hole, $1.6 \mathrm{~mm}$ cracks on each side)

Another aspect that needs to be taken into consideration during the design of an efficient SHM system is that of excitation frequency, which is the third dimension of the scatter cube presented in Figure 14b. The excitation frequency may play a very important role; referring to Figure 12a which studies the case of a normal incident beam, one notices that the excitation frequency may change the lobe pattern significantly (e.g., six lobes at $326 \mathrm{kHz}$, four lobes at $400 \mathrm{kHz}$, two lobes at $482 \mathrm{kHz}$, and seven lobes at $776 \mathrm{kHz}$.) The relative amplitude of the lobes in the pattern also changes. For a skew incident angle, this pattern changes again. Hence, the opportunity exists for analyzing various combinations of incident angles and frequencies in search for a best combination that would give an efficient SHM system design. Such a 
search is presented in Figure 16 for an $\mathrm{S} 0$ transmitter mode incident at various angles $\theta$ and over a wide frequency span up to $900 \mathrm{kHz}$. For each $f-\theta$ combination, the polar scatter plot was scanned azimuthally to identify the lobe with maximum amplitude and this maximum lobe amplitude was plotted in Figure 16.

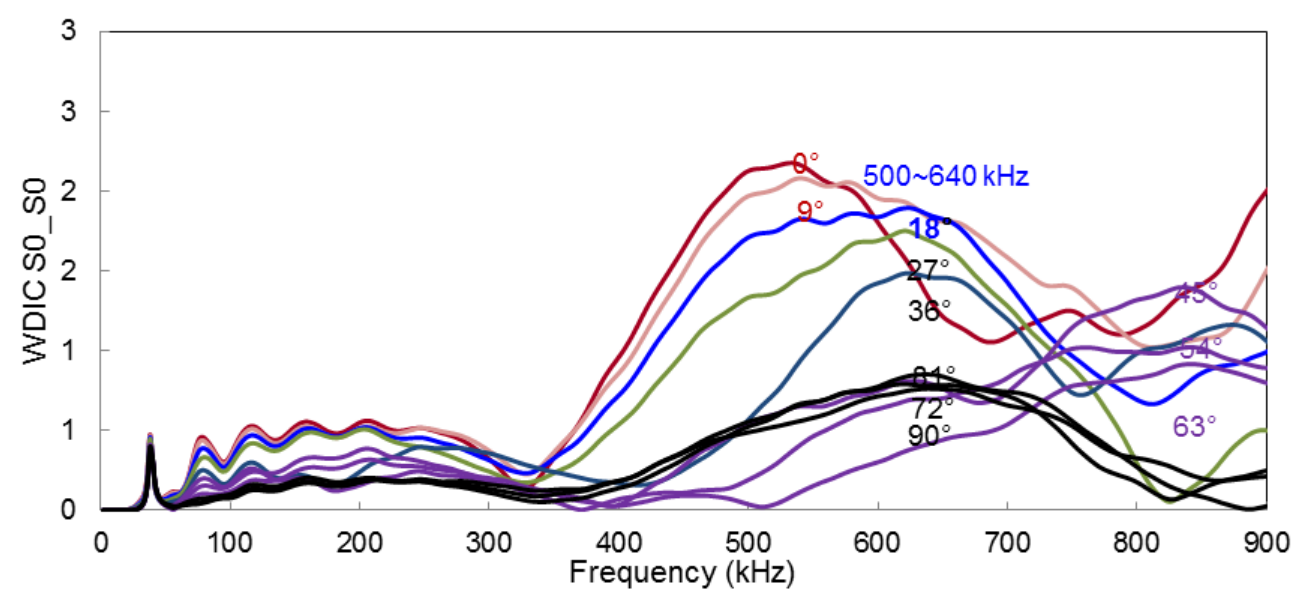

Figure 16: WDIC variation with frequency $f$ for S0 incident Lamb wave transmitted from various $\theta$ directions. WDIC was measured in a direction that was found to be the most sensitive for that particular $f-\theta$ combination

It is apparent from examination of Figure 16 that there are frequency-angle combinations that have a much larger scatter effect than others. In particular, the $500-640 \mathrm{kHz}$ range is very productive when the incidence angle is $\theta=18^{\circ}$. Whereas for other incidence, e.g., $\theta=72^{\circ}$, this frequency range would be quite inappropriate because the hole-scatter coefficients would be very small. Similar behavior, but with different values, was observed for A0 incident Lamb waves, as illustrated in Ref. [58].

\section{SUMMARY, CONCLUSIONS, SUGGESTIONS FOR FURTHER WORK}

\subsection{Summary}

This paper has reviewed our recent efforts in developing a predictive simulation of guided-wave structural health monitoring (SHM). After a short discussion of SHM scope, methods, and effect of confounding factors, the paper recalled the case of widespread fatigue damage (WFD) observed in long-service aircraft due to joining together of butterfly rivet-hole cracks in splice joints. In order develop a robust SHM system to address the WFD, one needs a fast and efficient forward problem that predicts the SHM signals that would be recorded for certain damage state, transducers layout, interrogation signals, ambient conditions, and other confounding factors.

The fast and efficient forward problem discussed in this paper is the hybrid global-local (HGL) approach in which a global analytical wave propagation formulation in the undamaged structure is combined with a local FEM discretization around the damage site. The HGL method analyzes the guided wave generation, propagation, interaction with damage, and reception during SHM with PWAS transducers. The HGL method is fast and efficient because it combines analytical solution in the global field with a local FEM solution that is small in size because it covers only the damage region and its vicinity. The waveform revealer (WFR) analytical framework was combined with wave-damage interaction coefficients (WDIC) calculated in the frequency-domain by a dense local FEM mesh with nonreflecting boundaries (NRB).

Both 1D and 2D situations were presented. Illustrative examples were given in the form of 1D scatter from a notch and 2D scatter from a rivet-hole butterfly crack encountered in practical WFD situation. The effect of transmitter and receiver angles as well as frequencies were examined.

\subsection{Conclusions}

It was found that fast and efficient predictive simulation of guided-wave structural health monitoring is possible via the HGL approach. The major conclusion of this work is that the HGL approach is highly efficient and hence recommended 
for the analysis of large structures in which damage has a localized nature. It would also be very useful in large-scale laboratory experiments.

The numerical examples constructed via WFR computational framework showed high dependency of the WDIC values on transmitter angle, receiver angle, and frequency. Certain optimal combinations of these parameters were identified and recommended for SHM system development and implementation.

The predictive methodology presented here can be used for (a) design of effective SHM systems; (b) performing sensitivity analysis towards a model-assisted determination of the probability of detection of the SHM system.

\subsection{Future work}

Suggestions for future work include (i) further development of the CMEP method for efficient analytical determination of the scatter field in $2 \mathrm{~d}$ situations; (ii) extension to the modeling of actual test coupons by the analytical introduction of boundary reflections; (iii) extension to acoustic emission studies; (iv) further development to cover guided-wave SHM of composite structures.

Though interesting, the work presented here is far from being complete. Several issues have still to be addressed, among which we distinguish (a) modeling of finite size specimens including boundary effects; (b) more efficient determination of WDIC values using a fast analytical approach; (c) performing experimental validation.

\subsubsection{Multimodal Guided Wave Propagation in a Finite-Size Specimen}

Experimental specimens are finite (Figure 3) whereas the analysis presented so far is performed in an infinite plate. For the analysis to be performed in finite-size specimens, the multimodal guided wave interaction with the specimen boundary must be analyzed. Our recently developed CMEP method [54] seems to be ideally suited for this analysis because it uses the full complement of Lamb wave modes (propagating, evanescent, and damped) that may be present in the plate. This analysis provides a fast and efficient calculation of reflection and conversion coefficients for any combination of multimodal Lamb waves impinging on a given specimen boundary at a given frequency [59]. A multitude of reflection and mode-conversion modes result from boundary interaction. Their number increases with frequency as more and more modes cross from the evanescent state into the propagating state.
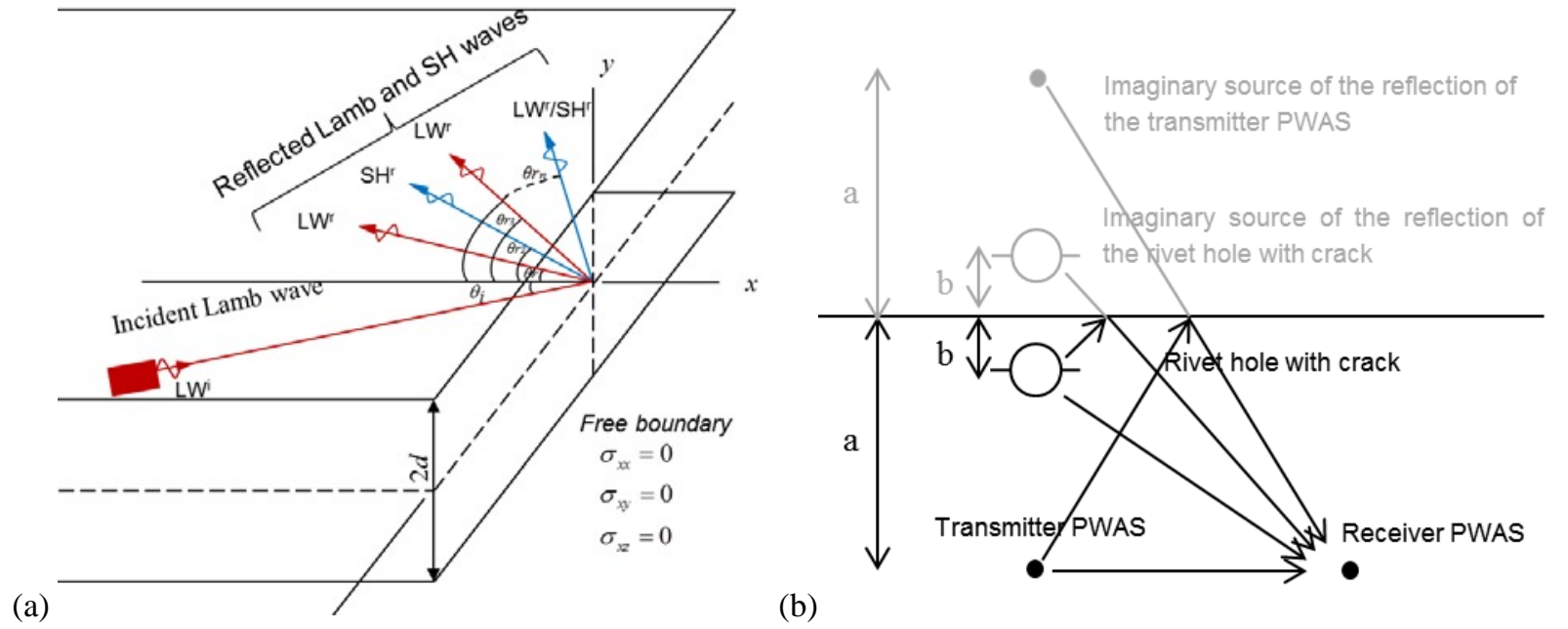

(b)

Figure 17 Issues to be address for modeling multimodal guided-wave propagation in a finite-size specimen: (a) reflection and mode conversion analysis at a plate boundary; (b) mirror image virtual sources

Another concept that could be exploited for analyzing multimodal guided wave propagation in a finite-size specimen is the method of mirrors [59]. A conceptual representation of this method is shown in Figure 17b; the domain of the specimen is extended beyond its physical boundaries by the addition of mirror "imaginary sources" representing the reflection and mode conversion effects. When applying the mirror image method to guided wave propagation, one will have to consider a multitude of imaginary source as corresponding to the multitude of Lamb wave modes resulting from the boundary interaction at any given frequency. 


\subsubsection{Analytical Calculation of Multimodal Guided Wave Interaction with Damage in 2D Geometry}

So far, the analysis of multimodal guided-wave interaction with damage in 2D geometry has been done only numerically using a local FEM mesh. However, our recently developed complex mode expansion with vector projection (CMEP) method [54] seems to be ideally suited for this analysis because it much faster and hence it allows for wide parameter searches. CMEP uses the full complement of Lamb wave modes (propagating, evanescent, and damped) that may be present in the plate. This analysis provides a fast and efficient calculation of reflection and conversion coefficients for any combination of multimodal Lamb waves impinging on a given specimen boundary at a given frequency.

\subsubsection{Experimental verification}

Simple coupon specimens (Figure 3) that are geometrically tractable, have been studied extensively and comparative results are readily available in the literature ([1], [60], [61], [62]). Multisite fatigue damage that appears in mechanicallyfastened splice joints and is related to butterfly cracks emanating from the fastener holes. Figure 18 shows a structural splice specimen to be used in our numerical and experimental studies. The splice joint will contain mechanical fasteners (tight-tolerance aerospace bolts) and cracks (Figure 2b).

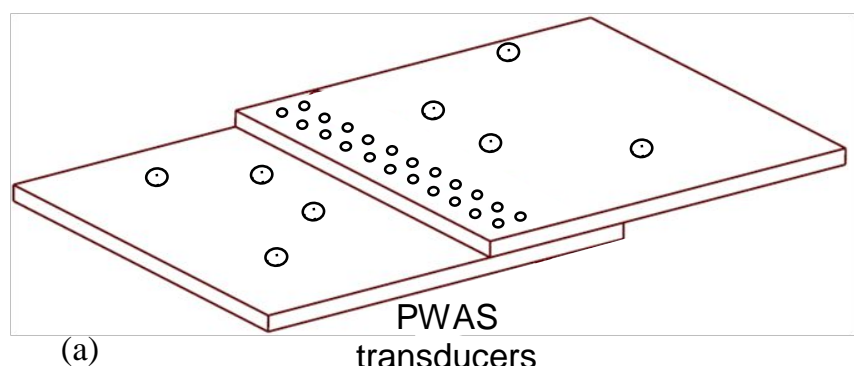

(a) transducers

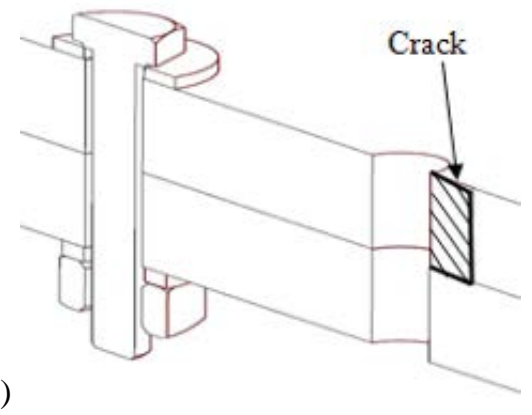

Figure 18: Realistic specimens to be studied in future work

\section{ACKNOWLEDGEMENTS}

Scientific credit is due to my former and current students and collaborators, ref. [8] [9] [17] [18] [49] [50] [51] [53] [52] [54] [56] [57] [58], [62], [63], [64], [65].

Financial support of Office of Naval Research grants, N000141110271 and N000141410655, Dr. Ignacio Perez technical representative; and of Air Force Office of Scientific Research FA9550-11-1-0133, Dr. David Stargel, Program Manager is gratefully acknowledged.

\section{REFERENCES}

[1] Chang, F.-K.; Janapati, V.; Kopsaftopoulos, F.; Lee, S. J.; Li, F. and Lonkar, K. (2014) "Quantification of Structural Health Monitoring for Damage Detection,” The 30th Aircraft Structural Integrity Program (ASIP), 2014 http://www.meetingdata.utcdayton.com/agenda/asip/2014/proceedings/presentations/P8198.pdf

[2] Worden, K; Inman, D J, "Modal Vibration Methods in Structural Health Monitoring," in Encyclopedia of Aerospace Engineering, Blockley, R S, W, Ed., ed: Wiley, 2010, pp. 1995-2004.

[3] Fan, W; Qiao, P (2011) "Vibration-based Damage Identification Methods: A Review and Comparative Study", Structural Health Monitoring - an International Journal, 10(1), 83-111, Jan 2011, doi: $10.1177 / 1475921710365419$

[4] Chang, F K (1995) "Built-In Damage Diagnostics for Composite Structures," 10th International Congress of Composite Materials ICCM-10, Canada, 1995, pp. 283-289

[5] Dalton, R. P.; Cawley, P.; Lowe, M. J. S. (2001) "The Potential of Guided Waves for Monitoring Large Areas of Metallic Aircraft Structure”, J. Nondestructive Evaluation, Vol. 20, pp. 29-46, 2001

[6] Raghavan, A.; Cesnik, C. E. S. (2007) "Review of guided-wave structural health monitoring", Shock and Vibration Digest, 2007. Vol. 39, No. 2, doi 10.1177/0583102407075428

[7] Chang, F-K (1998) "SMART layer - Built-in diagnostics for composite structures," 4th European Conference on Smart Structures and Materials, UK, 1998, pp. 777-781 
[8] Giurgiutiu, V.; Zagrai, A. N.; Bao, J. (2002) "Piezoelectric Wafer Embedded Active Sensors for Aging Aircraft Structural Health Monitoring”, Structural Health Monitoring - An International Journal, Sage Pub., Vol. 1, No. 1, July 2002, pp. 41-61

[9] Giurgiutiu, V.; Zagrai, A. N.; Bao, J. (2002) "Embedded Active Sensors for In-Situ Structural Health Monitoring of Thin-Wall Structures”, ASME Journal of Pressure Vessel Technology, Vol. 124, No. 3, August 2002, pp. 293302

[10] Giurgiutiu, V. (2014) Structural Health Monitoring with Piezoelectric Wafer Active Sensors, Elsevier Academic Press, 1032 pages, ISBN 9780124186910, 2014

[11] Yan, F; Royer, R L; Rose, J L (2010) "Ultrasonic Guided Wave Imaging Techniques in Structural Health Monitoring", Journal of Intelligent Material Systems and Structures, 21(3), 377-384, Feb 2010, doi: 10.1177/1045389x09356026

[12] Baravelli, E; Senesi, M; Ruzzene, M; De Marchi, L; Speciale, N (2011) "Double-Channel, Frequency-Steered Acoustic Transducer With 2-D Imaging Capabilities", IEEE Transactions on Ultrasonics Ferroelectrics and Frequency Control, 58(8), 1430 and 1706, Aug 2011, doi: 10.1109/tuffc.2011.2000

[13] Michaels, J. E.; Michaels, T. E. (2007) "Guided Wave Signal Processing and Image Fusion for In Situ Damage Localization in Plates”, Wave Motion, Vol. 44, pp. 482-492

[14] Kanajosyula, H.; Lissenden, C. J. (2013) "Analysis of annular phased array transducers for ultrasonic guided wave mode control,” Smart Materials and Structures, Vol. 22, pp. 085019

[15] Cho H; Cliff J. Lissenden, C. J. (2012) "Structural health monitoring of fatigue crack growth in plate structures with ultrasonic guided waves,” Struct. Health Mon. Int. J., Vol. 11, pp. 393-404

[16] Liu, L.; Yuan, F.-G. (2008) "Active damage localization for plate-like structures using wireless sensors and a distributed algorithm”, Smart Materials And Structures, Vol. 17 (2008) \#055022

[17] Zagrai, A.; Giurgiutiu, V. (2001) "Electro-Mechanical Impedance Method for Crack Detection in Thin Plates", Journal of Intelligent Material Systems and Structures, Vol. 12, No. 10, October 2001, pp. 709-71

[18] Giurgiutiu, V.; Zagrai, A. N. (2002) "Embedded Self-Sensing Piezoelectric Active Sensors for Online Structural Identification”, ASME Journal of Vibration and Acoustics, Vol. 124, January 2002, pp. 116-125

[19] Park, G.; Sohn, H.; Farrar, C. R.; Inman, D. J. (2003) "Overview of piezoelectric impedance-based health monitoring and path forward”, Shock and Vibration Digest, 2003, Vol. 35, pp 451-463

[20] Park, G; Inman, D J (2007) "Structural health monitoring using piezoelectric impedance measurements" Phil. Trans. Royal Soc. London, Series A, Vol. 365, No. 1851, Feb. 2007 373-392

[21] Aloha Airlines Flight 243 Explosive Decompression, 1988, https://en.wikipedia.org/wiki/Aloha_Airlines_Flight_243

[22] Southwest Airlines Flight 2294 Rapid Decompression, 2009, https://en.wikipedia.org/wiki/Southwest_Airlines_Flight_2294

[23] Southwest Airlines Flight 812 Rapid Decompression, 2011, https://en.wikipedia.org/wiki/Southwest_Airlines_Flight_812

[24] Hoggard, A. W.; Johnson, S. R. (2012) “Understanding the New Widespread Fatigue Damage Rule”, Aero Quarterly, Qtr_04, 2012, The Boeing Company

Press, http://www.boeing.com/commercial/aeromagazine/articles/2012_q4/pdfs/AERO_2012q4_article2.pdf

[25] Lindgren, E.; Stargel, D. (2012) "Foundational Challenges for Enhanced Damage Sensing”, European Workshop on Structural Health Monitoring, July 2012, Nantes, France

[26] K. Salama and C. K. Ling (1980), "The effect of stress on the temperature dependence of ultrasonic velocity," $J$. Appl. Phys., Vol. 51, No. 3, pp. 1505-1509, Mar. 1980

[27] Mazzeranghi, A; Vangi, D (1999) "Methodology for minimizing effects of temperature in monitoring with the acousto-ultrasonic technique", Experimental Mechanics, 39(2), 86-91, 1999, doi: 10.1007/bf02331110

[28] Weaver, R L; Lobkis, O I (2000) "Temperature dependence of diffuse field phase", Ultrasonics, 38(18), 491-494, 2000

[29] Lu, Y; Michaels, J E (2005) "A methodology for structural health monitoring with diffuse ultrasonic waves in the presence of temperature variations", Ultrasonics, 43(9), 717-731, 2005

[30] Andrews, J.P. (2007): “Lamb wave propagation in varying thermal environments”, MS Thesis, Air Force Institute of Technology, 2007

[31] Clarke, T; Simonetti, F; Cawley, P (2010) "Guided wave health monitoring of complex structures by sparse array systems: Influence of temperature changes on performance", Journal of Sound and Vibration, 329(12), 23062322, 2010 
[32] Croxford, A. J.; Moll, J.; Wilcox, P. D.; Michaels, J. E. (2010) "Efficient temperature compensation strategies for guided wave structural health monitoring,” Ultrasonics, Vol. 50, No. 4-5, pp. 517-528, Apr. 2010.

[33] A. Raghavan and C. E. S. Cesnik, "Effects of elevated temperature on guided-wave structural health monitoring," J. Intel. Mat. Syst. Str., Vol. 19, No. 12, pp. 1383-1398, Dec. 2008

[34] Michaels, J E; Lee, S J; Michaels, T E (2011) "Impact of applied loads on guided wave structural health monitoring", QNDE 2010 Vol. 30, AIP Conference Proceedings 1335, 2011, pp 1515-1522

[35] Sohn, H (2007) "Effects of environmental and operational variability on structural health monitoring," Phil. Trans. R. Soc. A, Vol. 365, No. 1851, pp. 539-560, Feb. 2007

[36] Lee, S. J.; Gandhi, N.; Michaels, J. E.; Michaels, T. E. (2011) "Comparison of the effects of applied loads and temperature variations on guided wave propagation”, QNDE 2010 Vol. 30, AIP Conference Proceedings 1335, 2011, pp 175-182

[37] Jiao, J P; Drinkwater, B W; Neild, S A; Wilcox, P D (2009) "Low-frequency vibration modulation of guided waves to image nonlinear scatterers for structural health monitoring", Smart Materials and Structures, Vol. 18 No. 6, doi 065006, 2009

[38] Ahmad, R; Kundu, T (2012) "Structural Health Monitoring of Steel Pipes under Different Boundary Conditions and Choice of Signal Processing Techniques", Advances in Civil Engineering, 2012, Vol 14, doi: $10.1155 / 2012 / 813281$

[39] Lindgren, E.; Medina, E.; Aldrin, J.; Buynak, C. (2011) “Demonstrating Capability Validation Protocol for In-situ Damage Detection”, ASIP Conference

2011, http://www.meetingdata.utcdayton.com/agenda/asip/2011/proceedings/presentations/P5121.pdf

[40] Goetschel, D.B.; Dong, S.B.; Muki, M. (1982) "A global local finite element analysis of axisymmetric scattering of elastic waves", Journal of Applied Mechanics, Vol. 49.

[41] Gengembre, N., Lhemery, A., Omote, R., Fouquet, T., Schumm, A. (2004) "A Semi-Analytical-FEM Hybrid Model for Simulating UT Configurations Involving Complicated Interactions of Waves with Defects”, CP700 Review of Quantitative Nondestructive Evaluation, Vol. 23, D. O. Thompson and D. E. Chimenti (Eds.), AIP 2004, pp. 74-80

[42] Lin, S.; Yamada, H.; Fukutomi, H.; Ogata, T. (2009) "Prediction of Received Signals in Ultrasonic Testing by Finite Element Method Combined with Geometrical Optics Theory”, CP1096 Review of Quantitative Nondestructive Evaluation, Vol. 28, D. O. Thompson and D. E. Chimenti (Eds.), AIP 2009, pp. 49-56

[43] Mahaut, S.; Lonne, S.; de Roumilly, L.; Cattiaux, G. (2006) "Validation of CIVA Simulation Tools for Ultrasonic Inspection in Realistic Configuration”, ECNDT September 25-29, 2006, Berlin, Germany, paper We.1.4.4 http://www.ndt.net/article/ecndt2006/topic 31.htm

[44] Lonne, S.; de Roumilly, L.; LeBer, L.; Mahaut, S.; Cattiaux, G. (2006) "Experimental Validation of CIVA Ultrasonic Simulation", $5^{\text {th }}$ International Conference on NDE in Nuclear Industry, San Diego, CA, USA, May 1012, 2006

[45] Chang, Z., and Mal, A. K. (1995) "A Global-Local Method for Wave Propagation Across a Lap Joint", in Numerical Methods in Structural Mechanics, Ju, J W (Ed.), ASME-AMD-Vol. 204, pp. 1-11, 1995

[46] Chang, Z.; Mal, A. (1999) "Scattering of Lamb waves from a rivet hole with edge cracks", Mechanics of Materials, Vol. 21, pp 197-204

[47] Mal, A. and Chang, Z. (2000) "A semi-numerical method for elastic wave scattering calculations", Geophysical Journal International, Vol. 143, pp. 328-334.

[48] Srivastava, A. (2009) "Quantitative structural health monitoring using ultrasonic guided waves", PhD dissertation, University of California San Diego.

[49] Gresil, M; Giurgiutiu, V (2013) "Time-Domain Hybrid Global--Local Concept for Guided-Wave Propagation with Piezoelectric Wafer Active Sensors", Journal of Intelligent Material Systems and Structures, Vol. 24, No. 15, pp. 1897-1911, 2013, doi: 10.1177/1045389x13486712

[50] Shen, Y (2014) “Structural Health Monitoring using Linear and Nonlinear Ultrasonic Guided Waves”, University of South Carolina PhD Dissertation, Aug. 2014

[51] Shen, Y.; Giurgiutiu, V. (2014) "WaveFormRevealer: An analytical framework and predictive tool for the simulation of multi-modal guided wave propagation and interaction with damage" Structural Health Monitoring An International Journal, Vol. 13, No. 5, pp. 491-511, online May 2014, doi: 10.1177/1475921714532986

[52] Shen, Y.; Giurgiutiu, V. (2015) "Effective non-reflective boundary for Lamb waves: theory, finite element implementation, and applications”, Wave Motion, Vol. 58, pp. 22-41 
[53] Shen, Y.; Giurgiutiu, V. (2016) "Combined analytical FEM approach for efficient simulation of Lamb wave damage detection”, Ultrasonics, Vol. 69, pp. 116-128

[54] Poddar, B.; Giurgiutiu, V. (2016) "Scattering of Lamb waves from a discontinuity: An improved analytical approach”, Wave Motion, Vol. 65 (2016), pp. 79-91

[55] Poddar, B (2016) "Physics Based Modeling of Guided Waves for Detection and Characterization of Structural Damage for NDE and SHM”, University of South Carolina PhD Dissertation, Aug. 2016

[56] Poddar, B.; Giurgiutiu, V. (2016) "Complex modes expansion with vector projection using power flow to simulate interaction of Lamb waves with notches”, Journal of the Acoustical Society of America, submitted manuscript \#JASA-01017

[57] Poddar, B.; Giurgiutiu, V. (2016) "Complex modes expansion with vector projection using power flow to simulate Lamb waves scattering from horizontal cracks and disbonds”, Journal of the Acoustical Society of America, Vol. 140, No. 3, pp. 2123-2133, September 2016

[58] Bhuiyan, Md Y; Shen, Y; Giurgiutiu, V (2017) "Interaction of Lamb waves with rivet hole cracks from multiple directions”, Proc IMechE Part C: J Mechanical Engineering Science, DOI: 10.1177/0954406216686996

[59] Achenbach, J D; Gautesen, A K; McMaken, H (1982) Ray Methods for Waves in Elastic Solids, Pitman, 1982Anon. (2015)

[60] Ihn, J-B (2003) "Built-in Diagnostics for Monitoring Fatigue Crack Growth in Aircraft Structures", PhD Dissertation, Stanford University, March 2003,

[61] Ihn, J.-B.; Chang, F.-K. (2008) "Pitch-catch Active Sensing Methods in Structural Health Monitoring for Aircraft Structures”, Structural Health Monitoring - An International Journal, Vol. 7, No. 1, pp. 5-19

[62] Bao, J; Giurgiutiu, V. (2015) "Acoustic Emission Event Characterization during Low Cycle Fatigue Experiments”, 57 ${ }^{\text {th }}$ Acoustic Emission Working Group Meeting, May 13-15, 2015, University of Illinois Chicago, http://www.aewg.org/aewg-57/2015.0429-AEWG-57-Booklet.pdf

[63] Yu, L (2006) "In-Situ Structural Health Monitoring Using Guided Lamb Wave Piezoelectric Wafer Active Sensors”, University of South Carolina PhD Dissertation, 2006

[64] Lin, B. (2010) "Power and Energy Transduction in Piezoelectric Wafer Active Sensors for Structural Health Monitoring: Modeling and Experiments”, University of South Carolina PhD Dissertation, 2010

[65] Poddar, B. (2016) "Physics Based Modeing of Guided Waves for Detection and Characterization of Structural Damage in NDE and SHM” University of South Carolina PhD Dissertation, Aug. 2016 